\title{
A residual correction method based on finite calculus
}

A residual correction method

Eugenio Oñate

International Center for Numerical Methods in Engineering (CIMNE), Universitat Politécnica de Catalunya, Barcelona, Spain

R.L. Taylor

Department of Civil and Environmental Engineering, University of California, Berkeley, USA

\section{O.C. Zienkiewicz}

Department of Civil Engineering, University of Wales, Swansea, UK J. Rojek

Institute of Fundamental Technological Research, Polish Academy of Sciences, Warsaw, Poland

Keywords Elasticity, Finite element method

Abstract In this paper, a residual correction method based upon an extension of the finite calculus concept is presented. The method is described and applied to the solution of a scalar convection-diffusion problem and the problem of elasticity at the incompressible or quasi-incompressible limit. The formulation permits the use of equal interpolation for displacements and pressure on linear triangles and tetrahedra as well as any low order element type. To add additional stability in the solution, pressure gradient corrections are introduced as suggested from developments of sub-scale methods. Numerical examples are included to demonstrate the performance of the method when applied to typical test problems.

\section{Introduction}

In a series of papers, Oñate et al. have introduced the concept of finite increment calculus (or finite calculus) method for providing a stabilization which is very effective (Oñate, 1998, 2000; Oñate and Arraez, 2002; Oñate and Garcia, 2001; Oñate and Manzan, 1999; Oñate et al., 1998, 2001). What we shall show in this paper is the problem of incompressible flow or elasticity which can reproduce very simply the ideas used in other stabilization processes already published or those recently in the process of introduction.

The concept of the finite calculus is to start the approximation from equations or a series of equations in which the size of the domain in which balance (or equilibrium) equations are established is finite and of the order of the finite spacing that will be used in the numerical solution. This physical concept is equivalent to the attempt of a discrete solution of a system of

Dedicated to the FEMClass of '42, (www.femclass42.com)
629

Received May 2002 Revised December 2002 Accepted January 2003 
$\mathrm{EC}$

$20,5 / 6$

630

equations that is of the form in which not only the original residual appears but also the derivatives of this residual, multiplied by the size of the increment used in the numerical process (which we will call $h$ ), appears. Thus, a very general statement can be made purely algebraically.

When using a numerical process for obtaining solution to a differential equation, it can be written in a much abbreviated form as

$$
r^{\alpha}=0
$$

where $\alpha$ may stand for one if a single equation is considered or in a more general system lists the whole set of governing equations being used. In the finite calculus, we solve a modified system of differential equations written as

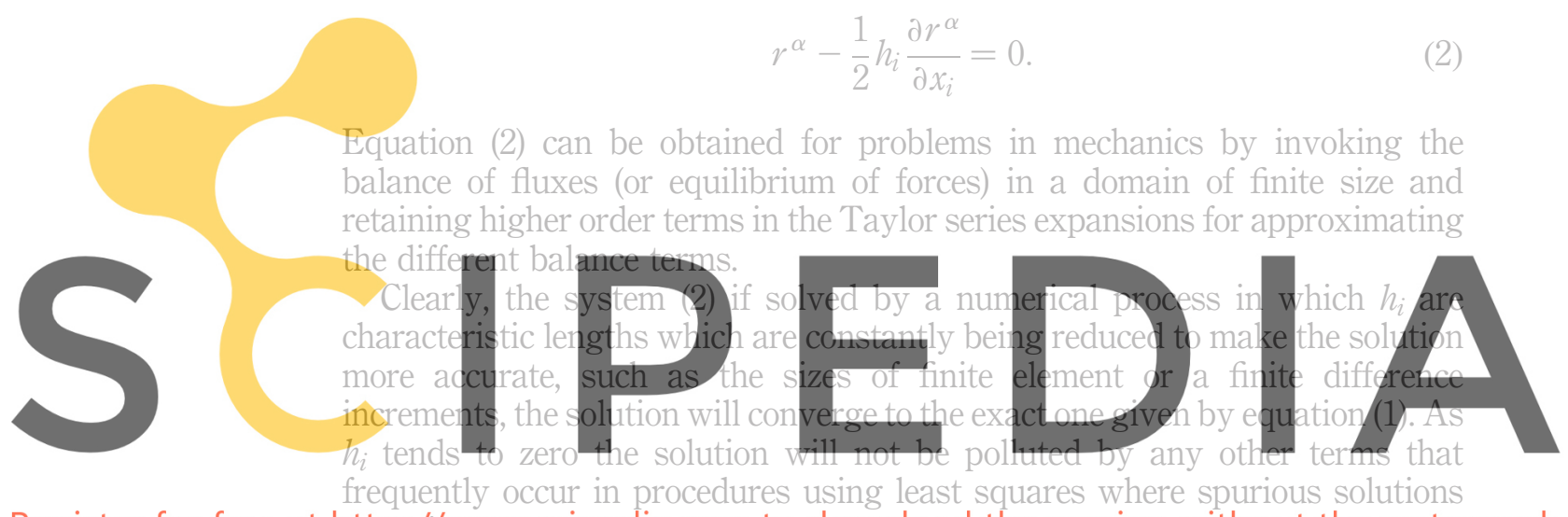
Register for free at hatpstimaswcscipedia.com to download the version without the watermark

It is at this stage important to observe that not all equations have to include additional terms involving $h_{i}$ as some of these where no stability problems arise can simply be omitted without destroying the general procedure. Indeed, the intelligent use of such omission should be applied by the investigator when dealing with a particular set of equations.

In Section 2, equation (2) is extended to a more general form of a residual correction method. We then consider the application of the extended process to the convection-diffusion equation with a scalar variable and show that it mimics the well known characteristic based Galerkin (CBS) or streamline upwinding process (Zienkiewicz and Taylor, 2000a).

We next consider the application of the process to linear elasticity in which incompressibility or near (quasi) incompressibility occurs. The case with full incompressibility is identical to the problem of Stokes flow and thus, the description is applicable to either process. It is well known that unless special precautions are taken the (near) incompressibility term, in numerical approximations in which displacement (velocity) and pressure are independently approximated, can lead to stability difficulties, with the well known Babuška-Brezzi limitations applicable (Babuška, 1973; Brezzi, 1974) or 
alternatively the mixed patch test (Zienkiewicz and Taylor, 1997, 2000b; Zienkiewicz et al., 1986) not being passed.

The application of a finite calculus process is introduced and shown to give alternative solution, including one which leads to the Brezzi-Pitkäranta (Brezzi and Pitkäranta, 1984) or Galerkin least squares (Hughes et al., 1989) result of adding a Laplacian term as well as an orthogonal sub-scale form (Codina, 2000; Codina and Blasco, 2000). These are obtained with very little manipulation. Indeed, once we derive these forms it will be clear that they are equally applicable once certain coefficients are identified.

The more complex process of time stepping introduced by Chorin (1967, 1968) and later generalized by Zienkiewicz and Codina (1995) and Zienkiewicz et al. (1995) can obviously be developed but here we shall delay its introduction to a later work.

\section{Theory}

As noted earlier, the solution of problems posed as differential equations is here expressed in a residual form as
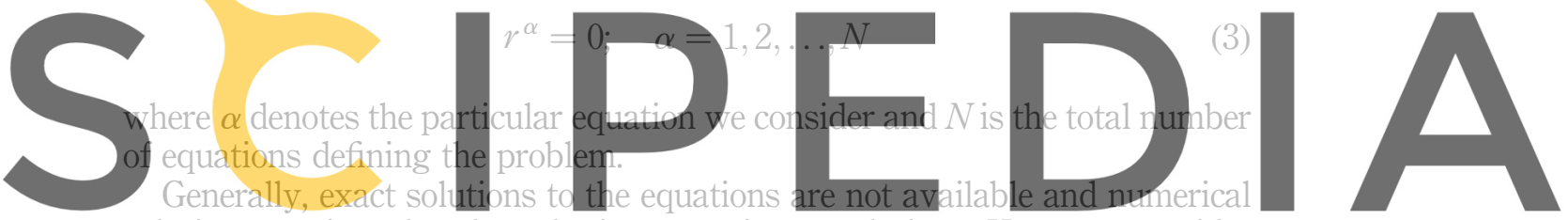

solutions are introduced to obtain approximate solutions. Here, we consider

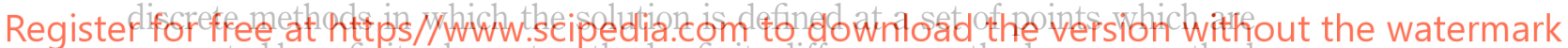
connected by a finite element method, a finite difference method, or any method in which some measure of their separation is defined by a parameter $h$ for each point or element.

For this case, starting from the set of modified equation (2) and after some manipulations we can extend the residual form to incorporate additional terms which vanish for an exact solution but give beneficial properties to the discrete formulation (Oñate et al., 2002). In the present work, we express the extended form as

$$
\hat{r}^{\alpha}=r^{\alpha}+\gamma_{i}^{\alpha \beta} h \frac{\partial r^{\beta}}{\partial x_{i}}=0
$$

where $i=1, \ldots, d$, with $d$ the space dimension of the problem being solved and a repeated index implies summation over the range of the index. Here, $h$ is a single characteristic length and the $\gamma_{i}^{\alpha \beta}$ are parameters which must be dimensionally consistent with the equation being solved $(\alpha)$ and the modifier equation $(\beta)$ added. For the case where $\alpha=\beta$ the parameter $\gamma_{i}^{\alpha \beta}$ is dimensionless. 
EC

$20,5 / 6$

632

The specification of a problem is completed by adding appropriate boundary (and for time dependent problems, initial) conditions which give a well posed form.

Here, we shall only consider a weak (weighted residual) form to construct approximate solutions. Accordingly, for equation (3) we use the solution form

$$
G\left(R^{\alpha}, r^{\alpha}\right)=\int_{\Omega} R^{\alpha} r^{\alpha} \mathrm{d} \Omega=0
$$

where $R^{\alpha}$ is an arbitrary conjugate variable to each equation. For equation (4) the solution form

$$
G\left(\hat{R}^{\alpha}, \hat{r}^{\alpha}\right)=\int_{\Omega} \hat{R}^{\alpha} \hat{r}^{\alpha} \mathrm{d} \Omega=0
$$

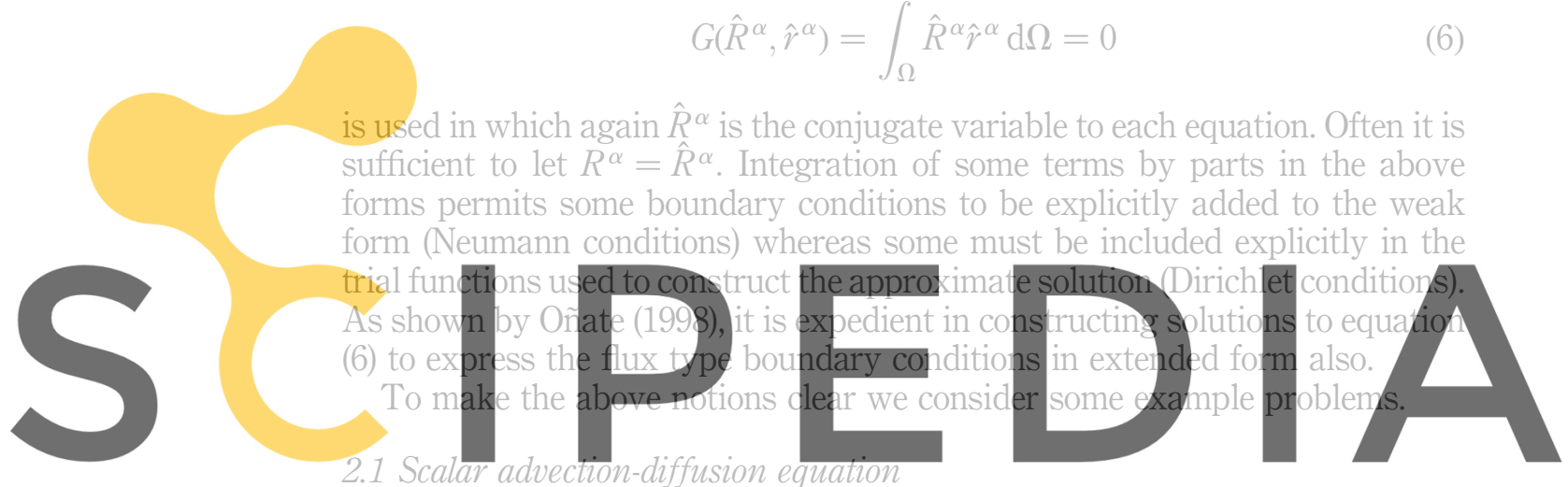

We first consider the scalar advection-diffusion equation given by

Register for free at https//www.scipedia.com to download the version without the watermark

$$
r=-a_{j} \frac{\partial \phi}{\partial x_{j}}+\frac{\partial}{\partial x_{j}}\left[k \frac{\partial \phi}{\partial x_{j}}\right]+Q=0 \text { in } \Omega
$$

in which $k$ is a positive diffusion parameter, $a_{j}$ are components of a specified vector, $Q$ is a given source term, $\phi$ is the dependent variable and $\Omega$ is the domain of the problem. The boundary conditions are given in terms of either Dirichlet,

$$
\phi=\bar{\phi} \quad \text { on } \Gamma_{1}
$$

or Neumann,

$$
q_{n}=-n_{j} k \frac{\partial \phi}{\partial x_{j}}=n_{j} q_{j}=\bar{q}_{n} \quad \text { on } \Gamma_{2}
$$

types where $n_{j}$ are components of an outward pointing normal to the boundary and $q_{j}$ are components of the diffusive flux.

2.1.1 Residual equation for solution. Here, there is only a single equation and we may write equation (4) in the simpler form 


$$
\hat{r}=r+\gamma_{i} h \frac{\partial r}{\partial x_{i}}=0
$$

indicating that only $d$ parameters need to be defined. A possible definition is to let

$$
\gamma_{i}=\gamma \frac{a_{i}}{|a|}
$$

633

where $|a|=\sqrt{a_{i} a_{i}}$ and $\gamma$ is a parameter to be selected.

2.1.2 Weak form of residual equation for numerical solution. Since there is only one equation, the arbitrary conjugate variable is related to $\phi$ and here we let

$$
\hat{R}=\delta \phi
$$

and a weak form for the extended problem is given as

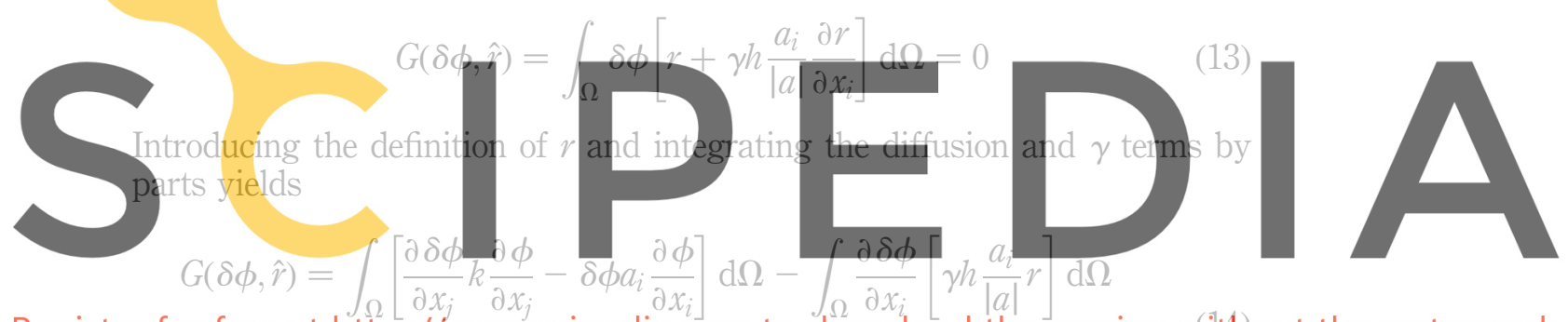

Register for free at https//www.scipedia.com to download the version withlout the watermark

$$
+\int_{\Gamma} \delta \phi\left[-n_{i} k \frac{\partial \phi}{\partial x_{i}}+n_{i} \gamma h \frac{a_{i}}{|a|} r\right] \mathrm{d} \Gamma=0
$$

In Oñate (1998), it is shown that the modified form of the Neumann boundary condition given by equation (14) can be anticipated a priori by using the concept of balance in a domain of finite size next to a boundary segment.The integrand of the boundary integral may be written as

$$
-n_{i} k \frac{\partial \phi}{\partial x_{i}}+n_{i} \gamma h \frac{a_{i}}{|a|} r=q_{n}+\gamma h \frac{a_{n}}{|a|} r
$$

where $a_{n}=n_{i} a_{i}$ is the component of the vector $a$ that is normal to the boundary. Here it is convenient to split the boundary into $\Gamma_{1}$ and $\Gamma_{2}$ and to consider an extended form of the Neumann boundary condition given by Oñate (1998).

$$
q_{n}+\gamma h \frac{a_{n}}{|a|} r=\bar{q} \quad \text { on } \Gamma_{2}
$$

together with $\delta \phi=0$ on $\Gamma_{1}$. With these additions the weak form becomes 
$\mathrm{EC}$

$20,5 / 6$

$$
\begin{aligned}
G(\delta \phi, \hat{r})= & \int_{\Omega}\left[\frac{\partial \delta \phi}{\partial x_{i}} k \frac{\partial \phi}{\partial x_{i}}-\delta \phi a_{i} \frac{\partial \phi}{\partial x_{i}}\right] \mathrm{d} \Omega-\int_{\Omega} \frac{\partial \delta \phi}{\partial x_{i}}\left[\gamma h \frac{a_{i}}{|a|} r\right] \mathrm{d} \Omega \\
& +\int_{\Gamma_{2}} \delta \phi \bar{q}_{n} \mathrm{~d} \Gamma=0
\end{aligned}
$$

634

It appears from the above construction that $C_{1}$ continuous interpolation is required in an approximate solution, whereas, in a normal Galerkin solution process, only $C_{0}$ interpolation is necessary. In the following, we shall assume only $C_{0}$ interpolation and ignore interface boundary terms (we could also argue that these terms will in fact tend to zero with $h$ or based on a residual which is zero for an exact solution).

It is instructive at this point to consider the form of the $\gamma$ term. To this end we note that

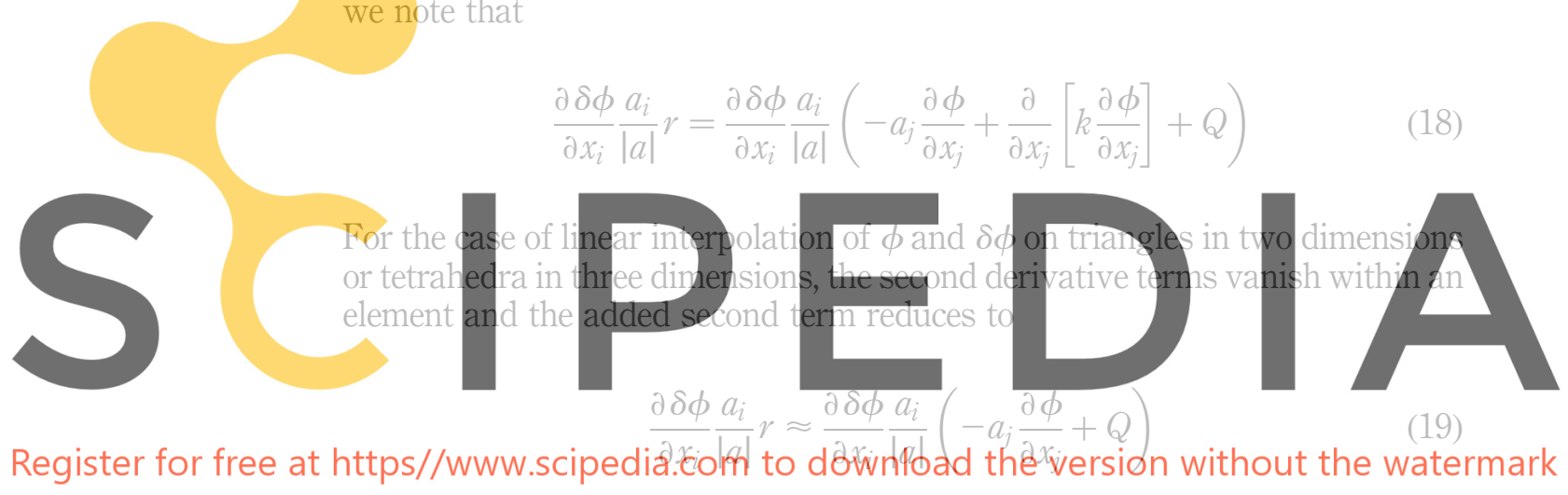

the first term on the right side being a conventional streamline diffusion type term and the latter a modification to any source term $Q$. The above is a well known process which mimics both the CBS (Zienkiewicz and Codina, 1995; Zienkiewicz et al., 1995) or the streamline upwind Petrov Galerkin (SUPG) (Brooks and Hughes, 1982) methods. Note that with the algebra used the value of $\gamma$ must be negative to add diffusion to the discrete problem (Oñate, 1998). Of course the optimal choice of $\gamma$ has to be decided.

Different alternatives for computing $\gamma$ taking advantage of the form of the modified governing equation (10) are given by Oñate (1998, 2000), Oñate and Arraez (2002), Oñate and Garcia (2001), Oñate and Manzan (1999) and Oñate et al. (1998). In practice the value of $\gamma$ generally follows that deduced in the mid 1970s (Christie et al., 1976; Zienkiewicz et al., 1977).

\subsection{Linear elasticity: incompressible limit behavior}

As a second example, we consider the problem of linear isotropic elasticity in which limits approach near incompressible behavior. As it is customary for this class of problems we first split the stress $\sigma_{i j}$ as 


$$
\sigma_{i j}=s_{i j}+\delta_{i j} p \quad \text { where } \quad p=\frac{1}{3} \sigma_{i i}
$$

In this form, $s_{i j}$ define deviator stresses and $p$ is the mean stress (pressure). Similarly, we split the strain $\varepsilon_{i j}$ as

$$
\varepsilon_{i j}=e_{i j}+\frac{1}{3} \delta_{i j} \varepsilon_{\mathrm{v}} \quad \text { where } \quad \varepsilon_{\mathrm{v}}=\varepsilon_{i i}
$$

635

In this form, $e_{i j}$ are deviator strains and $\varepsilon_{\mathrm{v}}$ is the (small strain) volume change. Using the above splits, we have the following systems:

Momentum equations. Ignoring effects of inertial loading, the balance of linear momentum describe the equilibrium behavior and are given by

$$
\frac{\partial s_{j i}}{\partial x_{j}}+\frac{\partial p}{\partial x_{i}}+b_{i}=0
$$

\section{where $b_{i}$ are body forces per unit of volume.}

Strain-displacement equations. The standard form for the infinitesimal

\section{rain-displacement rel}

in which $u_{i}$ are
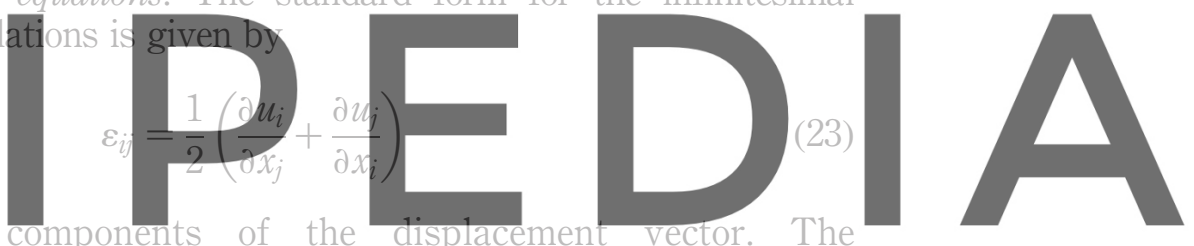

strain-displacement relations for the split form are given by

Register for free at https//www.scipedia.com to download the version without the watermark

$$
e_{i j}=\frac{1}{2}\left(\frac{\partial u_{i}}{\partial x_{j}}+\frac{\partial u_{j}}{\partial x_{i}}-\delta_{i j} \frac{2 \partial u_{k}}{\partial x_{k}}\right)
$$

for the deviator (isochoric) part and

$$
\varepsilon_{\mathrm{v}}=\frac{\partial u_{k}}{\partial x_{k}}
$$

for the volumetric part.

For an incompressible material, $\boldsymbol{\varepsilon}_{\mathrm{v}}$ will be zero and for a near (quasi) incompressible material it will be very small relative to the deviatoric part.

Stress-strain (constitutive) equations. The stress-strain relations for an isotropic linearly elastic material may be written as

$$
s_{i j}=2 G e_{i j}
$$

for the deviatoric part and

$$
p=K \varepsilon_{\mathrm{v}}
$$


EC

$20,5 / 6$

\section{6}

for the volumetric part. The two parameters $G$ and $K$ denote the shear modulus and bulk modulus, respectively. Note that the volumetric equation also may be written as

$$
\frac{1}{K} p=\varepsilon_{\mathrm{v}}
$$

and thus incompressible behavior implies $K=\infty$. Near or quasi incompressible behavior is characterized by $K / G \gg 1$. Using the definitions

$$
G=\frac{E}{2(1+\nu)} \text { and } \quad K=\frac{E}{3(1-2 \nu)}
$$

\section{near incompressible behavior is also given by $\nu \rightarrow 1 / 2$.}

Boundary conditions. The boundary conditions are given by specified displacements

$$
u_{i}=\bar{u}_{i} \quad \text { on } \Gamma_{1}
$$
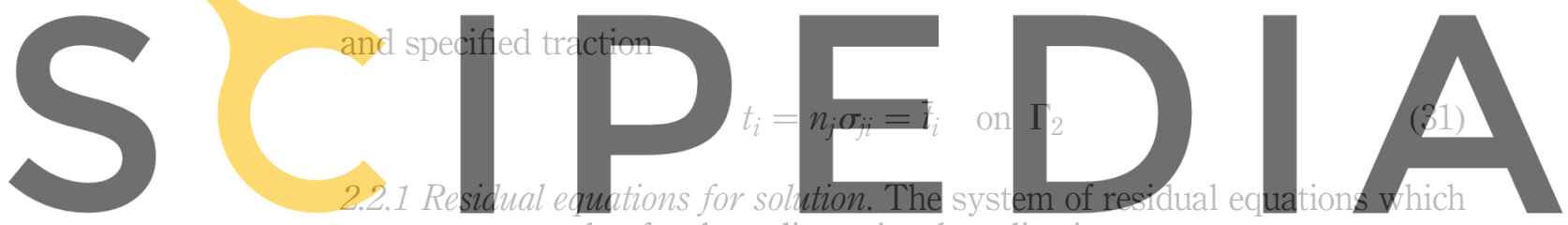

we propose to solve for three-dimensional applications are:

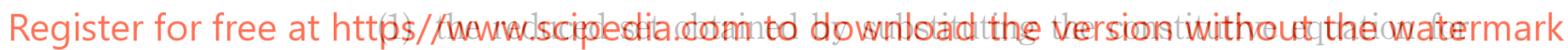
deviatoric stresses into the strain-displacement equations and then into the momentum equation; and

(2) the volumetric strain-displacement equation substituted into the constitutive equation for pressure-volume effects.

Accordingly, we will solve the resulting set of equations given by:

$$
\begin{aligned}
& r^{1}=\frac{\partial s_{j 1}\left(u_{k}\right)}{\partial x_{j}}+\frac{\partial p}{\partial x_{1}}+b_{1}=0 \\
& r^{2}=\frac{\partial s_{j 2}\left(u_{k}\right)}{\partial x_{j}}+\frac{\partial p}{\partial x_{2}}+b_{2}=0 \\
& r^{3}=\frac{\partial s_{j 3}\left(u_{k}\right)}{\partial x_{j}}+\frac{\partial p}{\partial x_{3}}+b_{3}=0 \\
& r^{4}=\frac{\partial u_{i}}{\partial x_{i}}-\frac{1}{K} p=0
\end{aligned}
$$


In a two-dimensional plane strain or axisymmetric problem we ignore the third equation and adjust ranges of the indices $i, j, k=1,2$. Plane stress gives no difficulties for any range of properties and may be considered using a pure displacement approach. In the above, we use the notation

$$
s_{i j}\left(u_{k}\right)=G\left[\frac{\partial u_{i}}{\partial x_{j}}+\frac{\partial u_{j}}{\partial x_{i}}-\delta_{i j} \frac{2}{3} \frac{\partial u_{k}}{\partial x_{k}}\right]
$$

637

to denote the effects of substitutions for the deviatoric behavior. We observe that equation (32) permits consideration of a solution when the bulk modulus $K$ is infinite (the incompressible limit). As is well known, only elements which pass the Brezzi-Babuška (Babuška, 1973; Brezzi, 1974) or mixed patch test (Zienkiewicz et al. 1986) lead to stable solutions and for low order elements these always involve different interpolation for $u_{k}$ and $p$. However, the use of the modified residual equations will allow accurate solution to be achieved even when the displacements $u_{k}$ and pressure $p$ are approximated within finite elements with linear interpolation on triangles or tetrahedra.

Before starting the solution process, we note that the momentum residuals

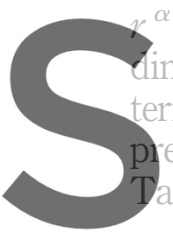

${ }^{\alpha}$ for $\alpha=1,2,3$ have dimensions $F / L^{3}$, where

mensionless. Thus, w

erms which restore ce

eviously for other ap profor

ylor, 2000b).

A simple construction
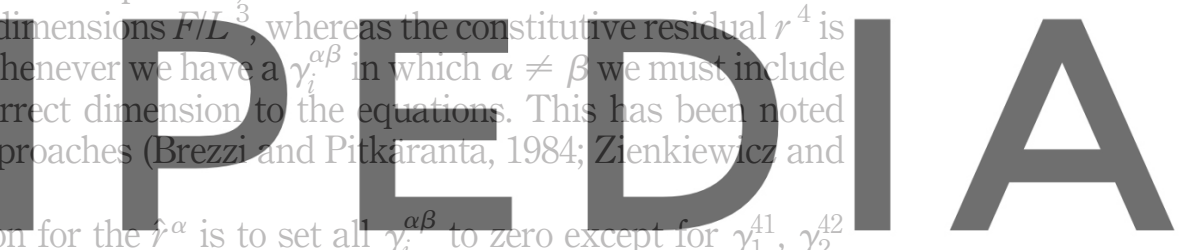

and $\gamma_{3}^{43}$. which we will set as

Register for free at https//www.scipedia.com to download the version without the watermark

$$
\gamma_{1}^{41}=\gamma_{2}^{42}=\gamma_{3}^{43}=\gamma \frac{h}{2 G}
$$

in which $\gamma$ is a single scalar variable to be chosen and the factor $h / G$ is chosen for dimensional considerations (we use $G$ as it remains bounded for all values of $K$ computed for $0<\nu \leq 0.5$ ). Thus, the residual equations we will use in our approximation are given by

$$
\hat{r}^{\alpha}=r^{\alpha} \quad \text { for } \alpha=1,2,3
$$

and

$$
\hat{r}^{4}=r^{4}+\gamma \frac{h^{2}}{2 G} \frac{\partial r^{i}}{\partial x_{i}}
$$

The modified pressure-volumetric strain equation (36) can also be derived by manipulating the original finite calculus forms of the equilibrium and pressure constitutive equations given by equation (2) (Oñate et al., 2002).

We expand the divergence of the first three residuals to give 
EC

$20,5 / 6$

$$
\frac{\partial r^{i}}{\partial x_{i}}=\frac{\partial^{2} s_{j i}}{\partial x_{i} \partial x_{j}}+\frac{\partial^{2} p}{\partial x_{i} \partial x_{i}}+\frac{\partial b_{i}}{\partial x_{i}}
$$

At this point there are different options we can pursue. The first is similar to

638 that introduced by Brezzi and Pitkäranta (1984) and extended to elasticity by Zienkiewicz and Taylor (2000b). In this approach we note (for constant $G$ and $K$ ) that

$$
\begin{aligned}
\frac{\partial^{2} s_{j i}}{\partial x_{i} \partial x_{j}} & =G\left(\frac{\partial^{3} u_{j}}{\partial x_{i} \partial x_{i} \partial x_{j}}+\frac{\partial^{3} u_{i}}{\partial x_{j} \partial x_{i} \partial x_{j}}-\frac{2}{3} \delta_{i j} \frac{\partial^{3} u_{k}}{\partial x_{k} \partial x_{i} \partial x_{j}}\right) \\
& =\frac{4 G}{3} \frac{\partial^{2}}{\partial x_{i} \partial x_{i}}\left(\frac{\partial u_{k}}{\partial x_{k}}\right) \\
& =\frac{4 G}{3 K} \frac{\partial^{2} p}{\partial x_{i} \partial x_{i}}
\end{aligned}
$$

which permits the derivative of the residual to be written as
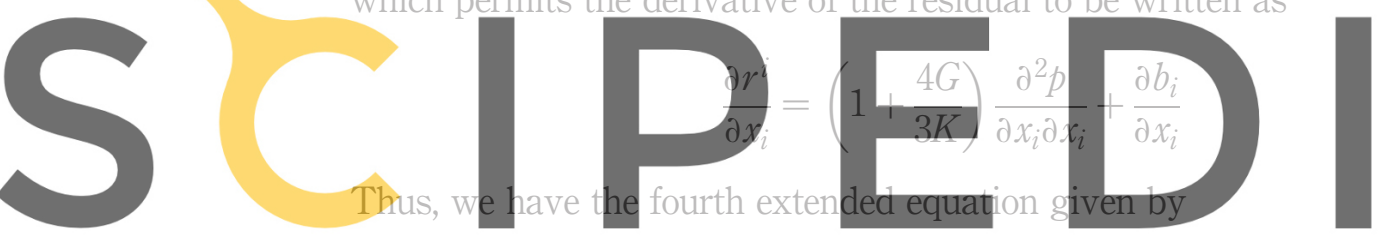

Register for free at https//www.scipêdia.cón tô downtoad the version without the wattermark

An aiternative approach to equation (40) represents the deviatoric and body force terms in the momentum equation by a new parameter set $\Pi_{i}$ in which

$$
\Pi_{i}=\frac{\partial s_{j i}\left(u_{k}\right)}{\partial x_{j}}+b_{i}=-\frac{\partial p}{\partial x_{i}}
$$

To use this approach, we must introduce the additional set of residual equations

$$
\begin{aligned}
& r^{5}=\frac{\partial p}{\partial x_{1}}+\Pi_{1}=0 \\
& r^{6}=\frac{\partial p}{\partial x_{2}}+\Pi_{2}=0 \\
& r^{7}=\frac{\partial p}{\partial x_{3}}+\Pi_{3}=0
\end{aligned}
$$


The introduction of $\Pi$ was deduced from an orthogonal sub-scale approach by Codina (2000) and Codina and Blasco (2000) and later employed by Chiumenti et al. (2002a, b) for elasticity and elasto-plasticity problems.

2.2.2 Weak form of residual equations for numerical solution. A Galerkin form to the equations may be constructed as

$$
G\left(\hat{R}^{\alpha}, \hat{r}^{\alpha}\right)=-\int_{\Omega} R^{i} r^{i} \mathrm{~d} \Omega+\int_{\Omega} \hat{R}^{4} \hat{r}^{4} \mathrm{~d} \Omega=0
$$

The first integral defines the statement on the momentum equation which we treat without modification and select

$$
\hat{R}^{i}=\delta u_{i} \quad \text { for } \quad i=1, \ldots, d .
$$

The second integral is the constitutive equation for pressure-volume which we consider modified to the extended form and set

$$
R^{4}=\delta p
$$

For the present, we do not consider which extended form will be used, however, we note that introduction of $\Pi_{i}$ will obviously require additional terms in equation (43).

After substitution of equation (32) and performing an integration by parts, the momentum terms become

$$
\begin{aligned}
G\left(\delta u_{i}, r^{i}\right)= & \int_{\Omega} \frac{1}{2}\left(\frac{\partial \delta u_{i}}{\partial x_{j}}+\frac{\partial \delta u_{j}}{\partial x_{i}}\right)\left[s_{i j}\left(u_{k}\right)+\delta_{i j} p\right] \mathrm{d} \Omega \\
& -\int_{\Omega} \delta u_{i} b_{i} \mathrm{~d} \Omega-\int_{\Gamma} \delta u_{i} t_{i} \mathrm{~d} \Gamma
\end{aligned}
$$

which we recognize as a standard Galerkin or virtual work form of the momentum equations.

Treating the second integral, we have the form

$$
G\left(\delta p, \hat{r}^{4}\right)=\int_{\Omega} \delta p\left[\frac{\partial u_{i}}{\partial x_{i}}-\frac{1}{K} p\right] \mathrm{d} \Omega+\int_{\Omega} \frac{\gamma h^{2}}{2 G} \delta p \frac{\partial r^{i}}{\partial x^{i}} \mathrm{~d} \Omega
$$

At this point we may either substitute using equation (39) or introduce the sub-scale terms $\Pi_{i}$. Introducing the Brezzi and Pitkäranta form and integrating by parts we obtain 
EC

$20,5 / 6$

$$
\begin{aligned}
G\left(\delta p, \hat{r}^{4}\right)= & \int_{\Omega} \delta p\left[\frac{\partial u_{i}}{\partial x_{i}}-\frac{1}{K} p\right] \mathrm{d} \Omega \\
& -\int_{\Omega} \frac{\gamma h^{2}}{2 G} \frac{\partial \delta p}{\partial x_{i}}\left[\left(1+\frac{4 G}{3 K}\right) \frac{\partial p}{\partial x_{i}}+b_{i}\right] \mathrm{d} \Omega \\
& +\int_{\Gamma} \delta p \frac{\gamma h^{2}}{2 G} n_{i}\left[\left(1+\frac{4 G}{3 K}\right) \frac{\partial p}{\partial x_{i}}+b_{i}\right] \mathrm{d} \Gamma
\end{aligned}
$$

\section{0}

Usually the last term is ignored in numerical computations. This act must be based on arguments concerning $h$ or $r_{i}$ tending to zero as, in general, the multiplier term is not zero. However, some controversy surrounds the results obtained by ignoring the term (Pierre, 1988, 1995).

An alternative is to introduce the sub-scale approximation defining $\Pi_{i}$ and directly integrate the residual term to obtain

$$
\begin{aligned}
G\left(\delta p, \hat{r}^{4}\right)= & \int_{\Omega} \delta p\left[\frac{\partial u_{i}}{\partial x_{i}}-\frac{1}{K} p\right] \mathrm{d} \Omega-\int_{\Omega} \frac{\gamma h^{2}}{2 G} \frac{\partial \delta p}{\delta x_{i}}\left[\frac{\partial p}{\partial x_{i}}+\Pi_{i}\right] \mathrm{d} \Omega \\
& +\int_{\Gamma} \delta p \frac{\gamma h^{2}}{2 G} n_{i}\left[\frac{\partial p}{\partial x_{i}}+\Pi_{i}\right] \mathrm{d} \Gamma
\end{aligned}
$$

In this form, the boundary integral again involves $h$ and a residual which permits it to be dropped without recourse to material behavior. Indeed, we may introduce any constitutive equations for other material behavior (e.g. visco-elasticity, elasto-plasticity, etc.) without altering the definition of $\Pi_{i}$.

Combining the above two terms and introducing a split on the boundary for displacement and traction types we can write the final weak form for the solution of the elasticity problem as

$$
\begin{aligned}
G\left(\delta u, \delta p, \hat{r}^{\alpha}\right)= & \int_{\Omega} \frac{1}{2}\left(\frac{\partial \delta u_{i}}{\partial x_{j}}+\frac{\partial \delta u_{j}}{\partial x_{i}}\right)\left[s_{i j}\left(u_{k}\right)+\delta_{i j} p\right] \mathrm{d} \Omega \\
& -\int_{\Omega} \delta u_{i} b_{i} \mathrm{~d} \Omega-\int_{\Gamma_{2}} \delta u_{i} \bar{t}_{i} \mathrm{~d} \Gamma \\
& +\int_{\Omega} \delta p\left[\frac{\partial u_{i}}{\partial x_{i}}-\frac{1}{K} p\right] \mathrm{d} \Omega-\int_{\Omega} \frac{\gamma h^{2}}{2 G} \frac{\partial \delta p}{\partial x_{i}} r^{i} \mathrm{~d} \Omega=0
\end{aligned}
$$

where $r^{i}$ is either deduced from equation (39) or is given by the $\Pi_{i}$ form of the momentum equations expressed in equation (42). In the latter case, it is necessary to introduce a weighted residual form for the equations to provide a solution for the added variables. Two forms have been proposed for this 
projection. The first based on the orthogonal sub-scale argument adds the equations as

$$
G=\int_{\Omega} \frac{\gamma h^{2}}{2 G}\left[\delta \Pi_{1} r^{5}+\delta \Pi_{2} r^{6}+\delta \Pi_{3} r^{7}\right] \mathrm{d} \Omega=0
$$

where we have set $R^{i+4}=\delta \Pi_{i}$. The second form merely uses

A residual correction

method

641

$$
G=\int_{\Omega}\left[\delta \Pi_{1} r^{5}+\delta \Pi_{2} r^{6}+\delta \Pi_{3} r^{7}\right] \mathrm{d} \Omega=0
$$

thus avoiding the $\gamma$ term completely. For the elasticity problem the form given by equation (51) leads to fully symmetric equations which is an advantage if the equations are solved as one set. If, however, a split of the equations is used and the solution for equation (50) is first obtained with the $\Pi_{i}$ set at their previous value (i.e. zero at the first iteration) followed by solution of either equation (51) or (52) little is gained by introducing the $\gamma$ weighting.

Note that a typical term in equation (51) or (52) is given by

$$
\delta \Pi_{1} r^{5}=\delta \Pi_{1}\left[\frac{\partial p}{\partial x_{1}}+\Pi_{1}\right]
$$

Thus, treating $R^{5}$ as an arbitrary (virtual) $\Pi_{1}$ will lead to a mass type matrix in the numerical solution process (Brooks and Hughes, 1982; Codina, 2000; Oñate et al., 2002). The idea of projecting values by what is essentially a least squares method is not new. In the context of stress projections it was used by Brauchli and Oden (1971). Cantin et al. (1978) and Loubignac et al. (1978) used a similar approach to build improved stress results from a displacement solution and in a mixed approximation and solution context this was extended by Zienkiewicz et al. (1985a, b). As noted earlier, the approximate recovery of the pressure gradients is not unique and other schemes can be considered (e.g. a superconvergent patch recovery (SPR) projection as introduced by Zienkiewicz and Zhu (1992).

\section{Numerical examples}

The above formulation has been implemented in the general purpose finite element program FEAP which is described by Taylor, (2003) and Zienkiewicz and Taylor (2000b, c). This formulation permits solutions using either the fully monolithic algorithm in which the displacements, pressures and pressure gradient projections are computed simultaneously or using a split algorithm in which the displacements and pressures are computed separately from the pressure projection values and iterative improvements are used (Oñate $e t$ al., 2002). 
EC

642
$20,5 / 6$

\subsection{Cook problem}

We first consider the problem shown in Figure 1 known as the Cook problem. The original problem was given as a plane stress problem; however, to test the performance of elements in a quasi incompressible state it has been given as a plane strain problem. The properties are taken as shear modulus with a value of $G=375$ and Poisson ratio with a value of $v=0.4999$.

The remaining geometric factors are shown in Figure 1. Meshes of quadrilaterals and linear triangles are used with 2, 5, 10, 20 and 50 elements on each side of the mesh. The meshes for five elements per side are shown in Figure 2. To illustrate the effects of locking for this problem we also consider

Figure 1.

Cook problem description
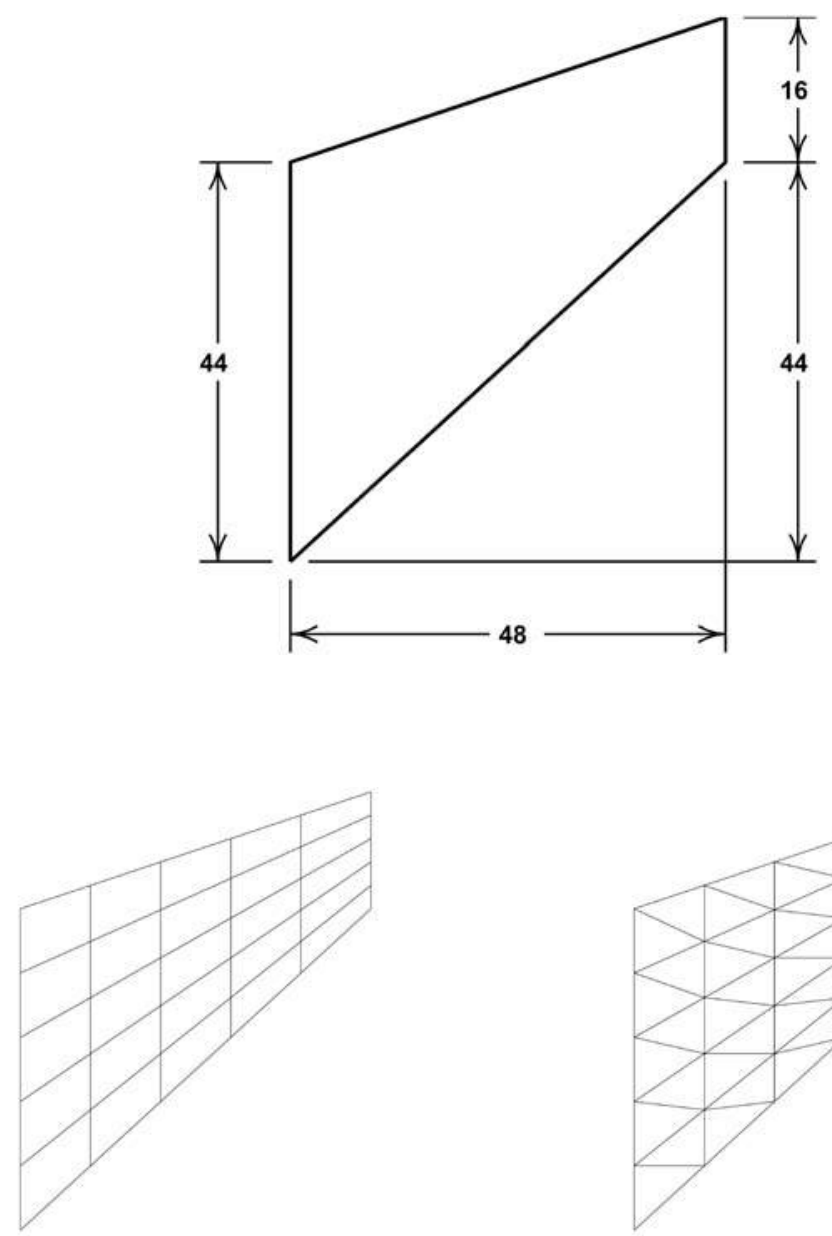

(a) $5 \times 5$ Quadrilateral Mesh

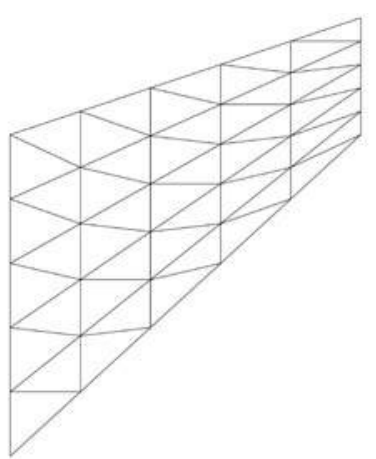

(b) $5 \times 5$ Triangular Mesh
Figure 2.

Cook problem meshes: plane strain 
standard displacement models employing linear triangles and four-node quadrilateral elements.

The displacements on the left boundary are fully restrained and a uniformly distributed shear load with unit intensity is applied to the right boundary.

In Figure 3, we present results for the vertical displacement at the top of the right boundary. The left figure presents results for the standard displacement formulation (Q1), the mixed Q1P0 element described by Simo et al. (1985) and the enhanced assumed strain element (Q1Enh) given by Simo and Rifai (1990). In the right part of the figure, we present results for triangles using the standard displacement model (T1), the mixed displacement pressure

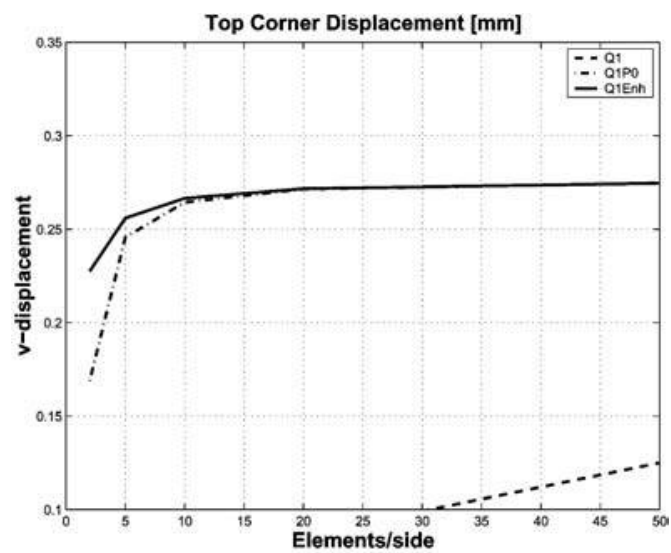

(a) Quadrilateral Results

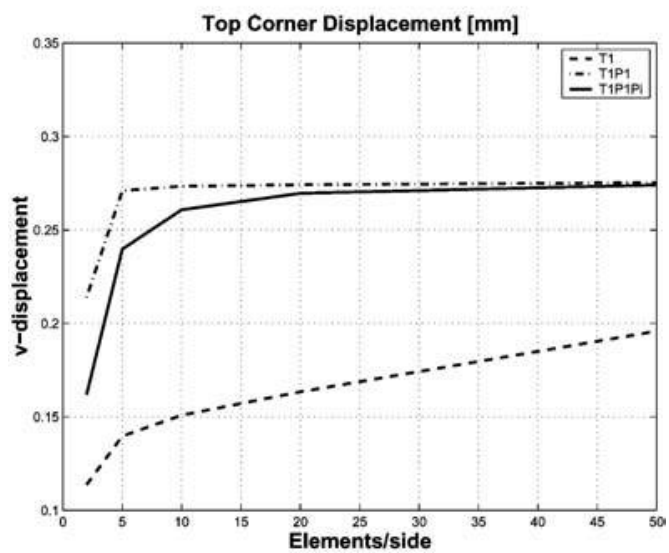

Figure 3. Cook problem: vertical displacement at top

(b) Triangular Results corner 
EC

$20,5 / 6$

644

Figure 4.

Cook problem: vertical displacement at top corner form (T1P1) and the new form (T1P1Pi). The results for the T1P1 element where both displacements and pressure are defined using continuous linear interpolation on each triangle are in fact identical to many other forms (Zienkiewicz and Taylor, 2000b) and occur as the first solution in the iterative split form. It is evident that adding the pressure projection does not improve the accuracy for the displacement at this point. Indeed, the effect is more evident in the pressure distribution as we shall show in subsequent examples.

In Figure 4, we compare the results for the Q1Enh and T1P1Pi elements. It is remarkable that the triangle performs quite well compared with the quadrilateral element, which in fact is excellent in both quasi-incompressible and bending applications.

In Figures 5 and 6, we present the same type of results as just described for the horizontal displacement at the top. In addition, in Figures 7-10, we show results for the bottom corner displacement components. Generally, behavior of results are similar to those at the top. However, we note that the displacements at these two points converge in a more monotonic manner using the formulation including the pressure projection variables $\Pi_{i}$.

\subsection{Tension strip with slot}

The second problem considered is a confined strip with a central slot with circular ends. The problem is loaded by a uniform axial load at the top and bottom and has geometric properties as shown in Figure 11. The material properties are: Young's modulus $(E)$ of 24 and Poisson ratio $(\nu)$ of 0 . 49999995. The analysis is performed in plane strain to again give a quasi-incompressible response. This problem has no infinite stress at any point and thus provides a check on the triangular elements to perform well over the entire problem.

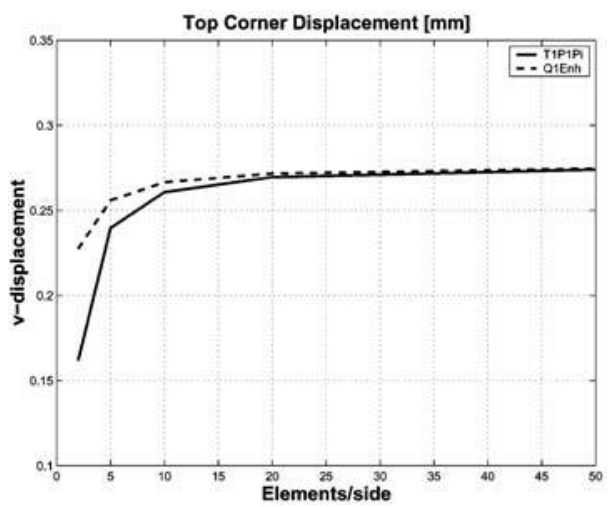




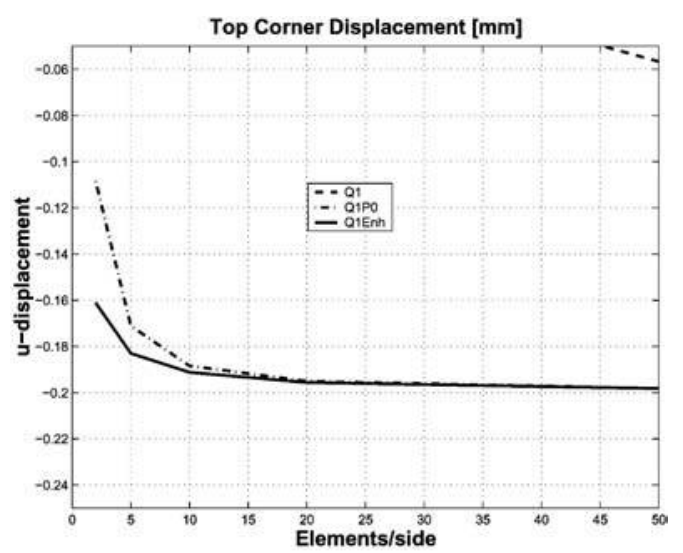

A residual correction method

(a) Quadrilateral Results

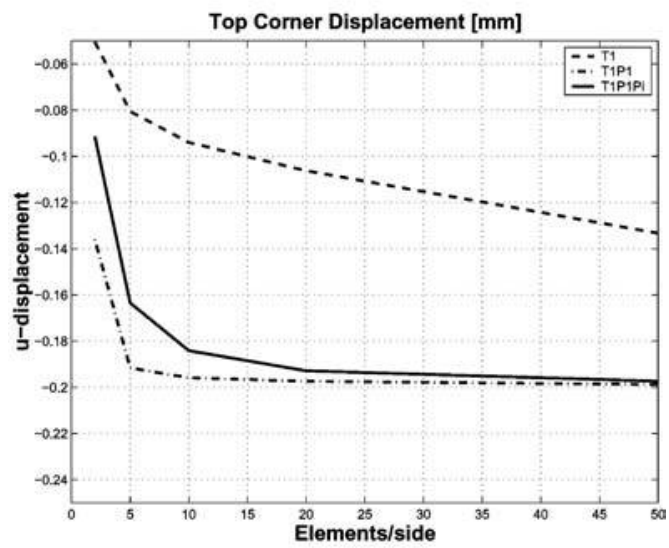

Figure 5.

Cook problem: horizontal displacement at top

(b) Triangular Results corner

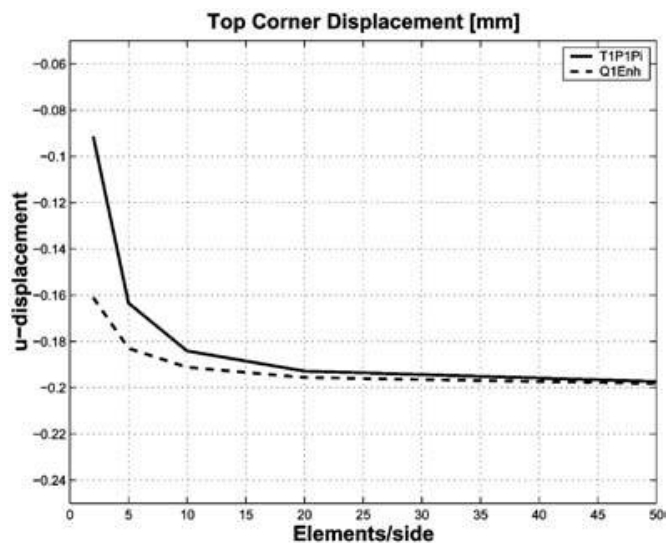

Figure 6. Cook problem: horizontal displacement at top corner 
EC

20,5/6

\section{6}

Figure 7.

Cook problem: vertical displacement at bottom corner
Figure 8.

Cook problem: vertical displacement at bottom corner

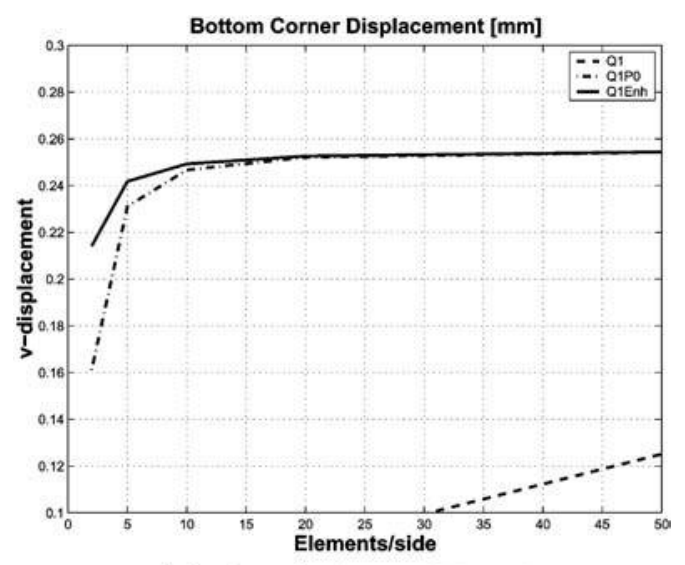

(a) Quadrilateral Results

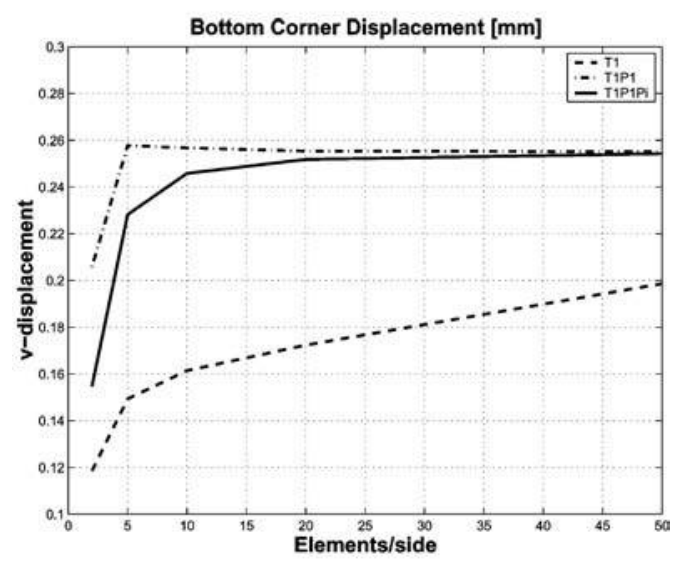

(b) Triangular Results

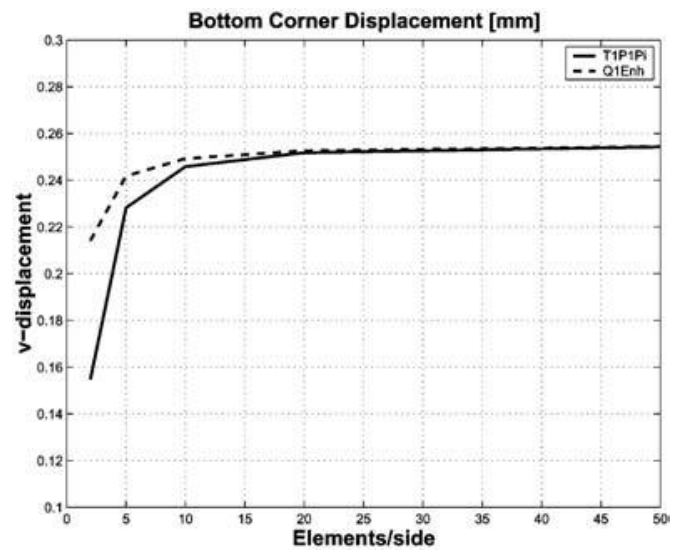




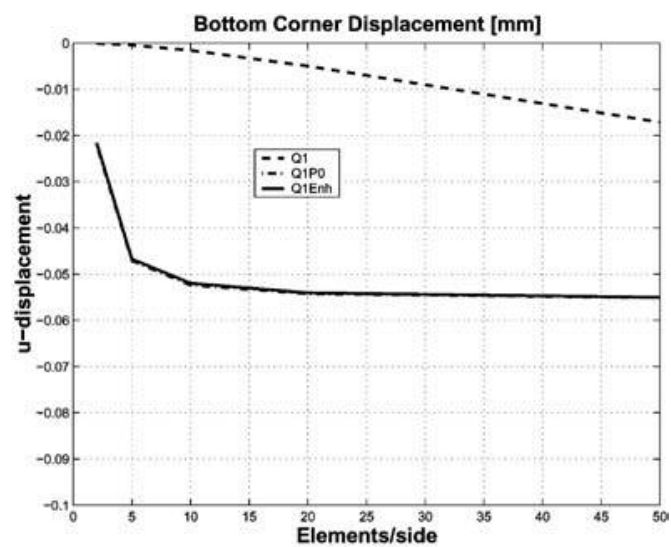

A residual correction method

(a) Quadrilateral Results

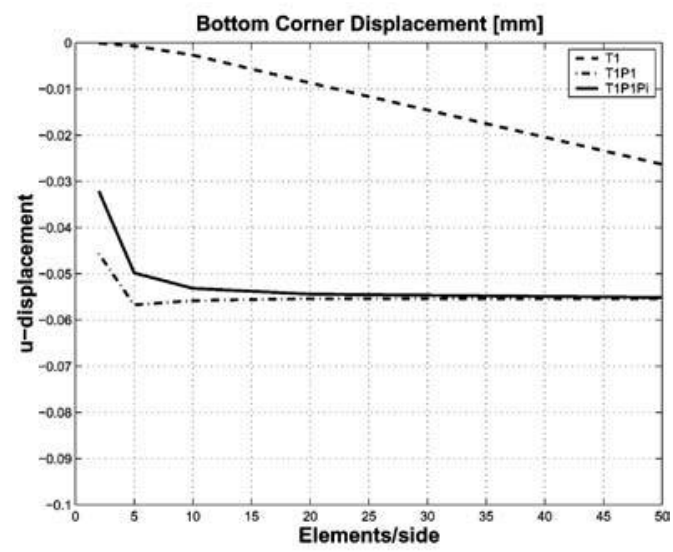

Figure 9.

Cook problem: horizontal displacement at bottom

(b) Triangular Results corner

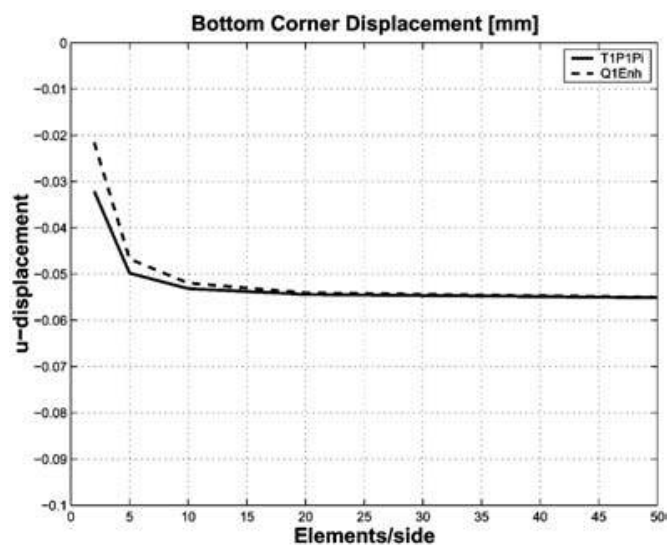

Figure 10. Cook problem: horizontal displacement at bottom corner 
EC

$20,5 / 6$

648

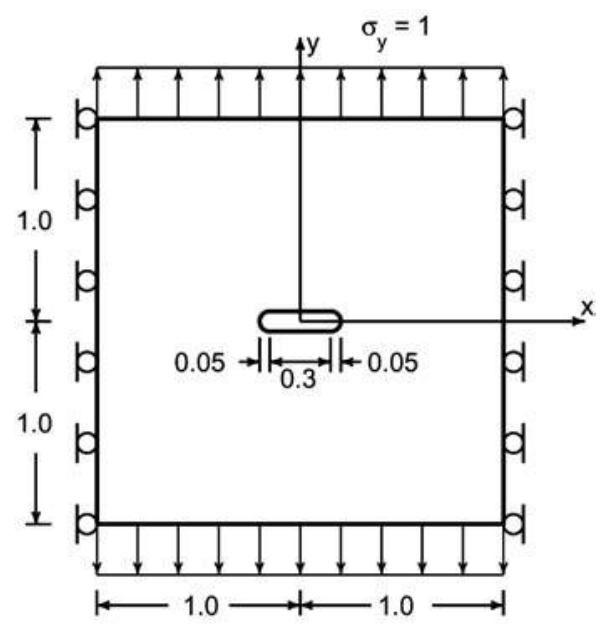

(a) Problem description

Figure 11.

Region and mesh used for slotted tension strip

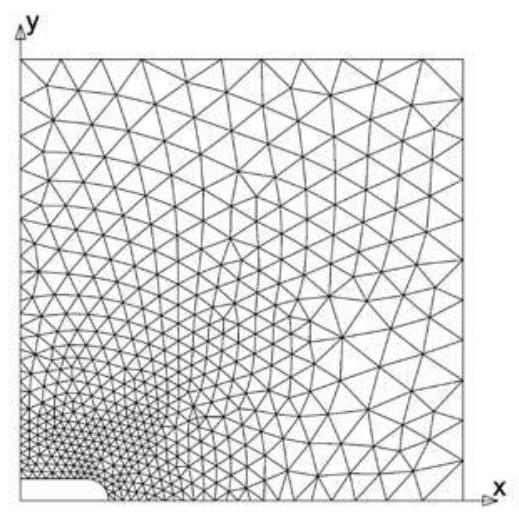

(b) Mesh for quadrant

The distribution of pressure along the axes is presented in Figure 12 for cases with the $\Pi_{i}(\mathrm{~T} 1 \mathrm{P} 1 \mathrm{Pi})$ and for the Brezzi-Pitkäranta form (T1P1). Both are free of oscillations, however, the form which includes the $\Pi_{i}$ modification produces answers which are in closer agreement with other methods (Zienkiewicz and Taylor, 2000b).

\subsection{Driven cavity}

We consider the problem of a square region in which the top is displaced horizontally by a unit displacement. This is a standard problem in fluid mechanics known as the driven cavity problem in which a velocity is specified at 


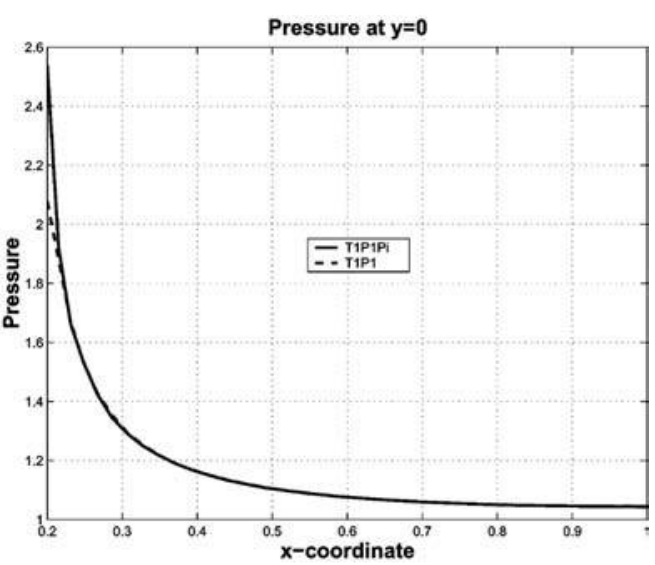

(a) Pressure on $x$-axis

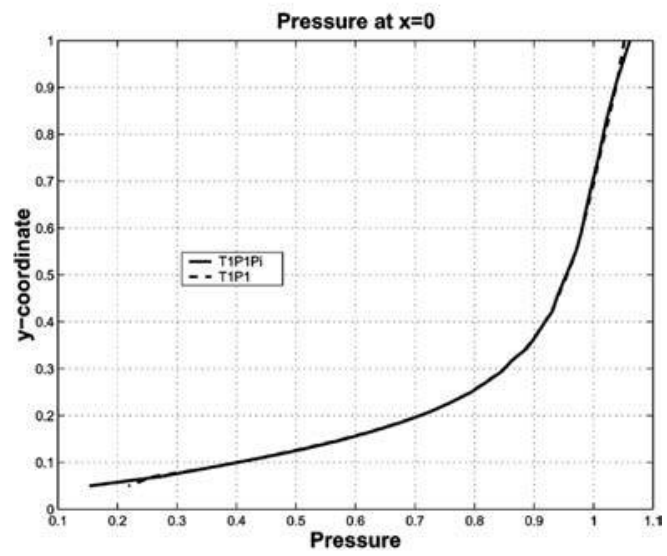

(b) Pressure on y-axis
A residual correction method

649
Figure 12. Pressure distribution on axes of slotted tension strip

the top instead of a displacement (Oñate and Garcia, 2001). The cavity has a unit length for both the horizontal and vertical sides. The material properties are Young's modulus, $E$, of 3 and Poisson ratio, $\nu$, of 0.49999995 . This leads to shear modulus of near unity, a ratio of the bulk to shear modulus $(K / G)$ approximately equal to $10^{7}$ and, thus, represents a quasi-incompressible solid. To represent the driven cavity, all boundaries have zero displacement in both the normal and tangential directions except the top face which has a unit horizontal displacement at all nodes except the corner ones which are set to zero.

For the solution reported here, the pressure at the center of the bottom face is set to zero. The problem is solved using different meshes ranging from ten 
EC

$20,5 / 6$

650 elements per side to 40 elements per side. The $10 \times 10$ mesh is shown in Figure 13 .

To illustrate the effects of the sub-scale $\Pi_{i}$ terms, we plot the pressure along the horizontal centerline in Figure 14. The curve labeled T1P1 ignores the I terms in all equations and thus leads to a form which includes only the displacement $\mathbf{u}$ and pressure $p$ at each node. In this form, the equations are identical to that introduced originally by Brezzi and Pitkäranta (1984).
Figure 13.

The $10 \times 10$ mesh of triangles
Figure 14.

Pressure on horizontal centerline for $10 \times 10$ mesh

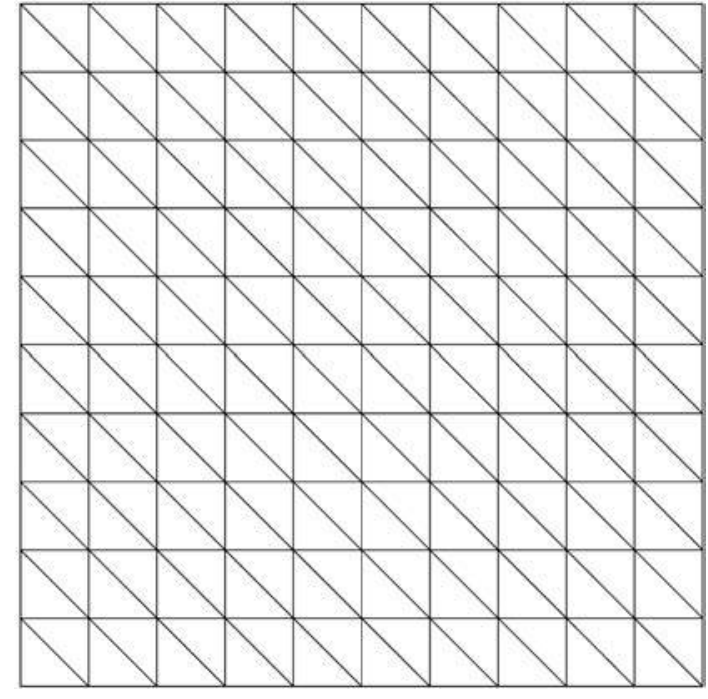

Cavity: Pressure at $\mathrm{y}=0$

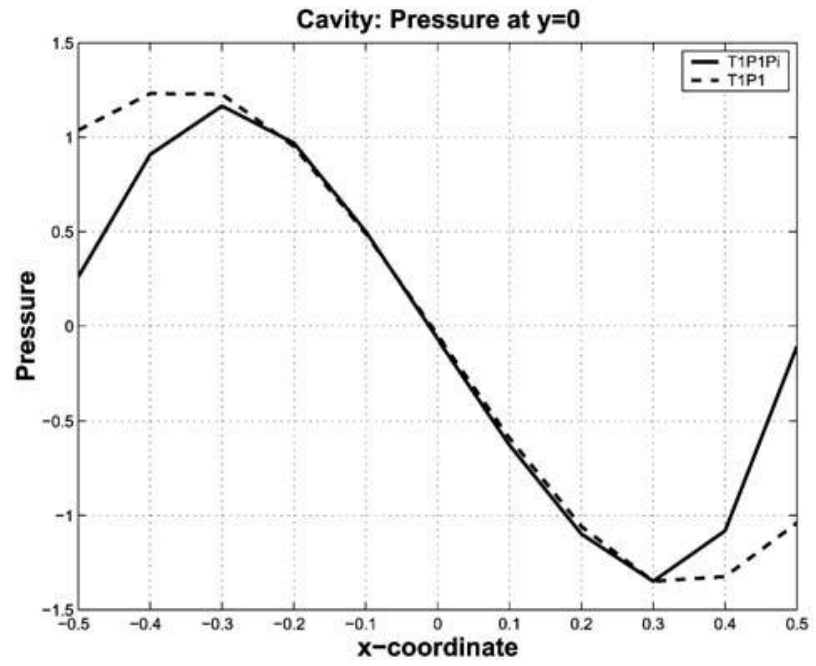


In Figure 15 we show the same result for a $40 \times 40$ mesh. These results have been computed with the monolithic algorithm in which all five degrees of freedom $\left(u_{1}, u_{2}, P, \Pi_{1}\right.$ and $\left.\Pi_{2}\right)$ are included in a single solution. The value for stabilization array is set as $\tau=\gamma h^{2} /(2 G) \mathbf{I}$ where $h^{2}$ is taken as twice the area of each triangle and $\gamma=0.75$ as given by a finite calculus derivation (Oñate et al., 2002).

The above solution was repeated using the iterative solution in which the displacement and pressure are solved separately from the $\Pi$ variables. Figures 16 and 17 show the results for iterations 1,3 , and 5 . The result for the
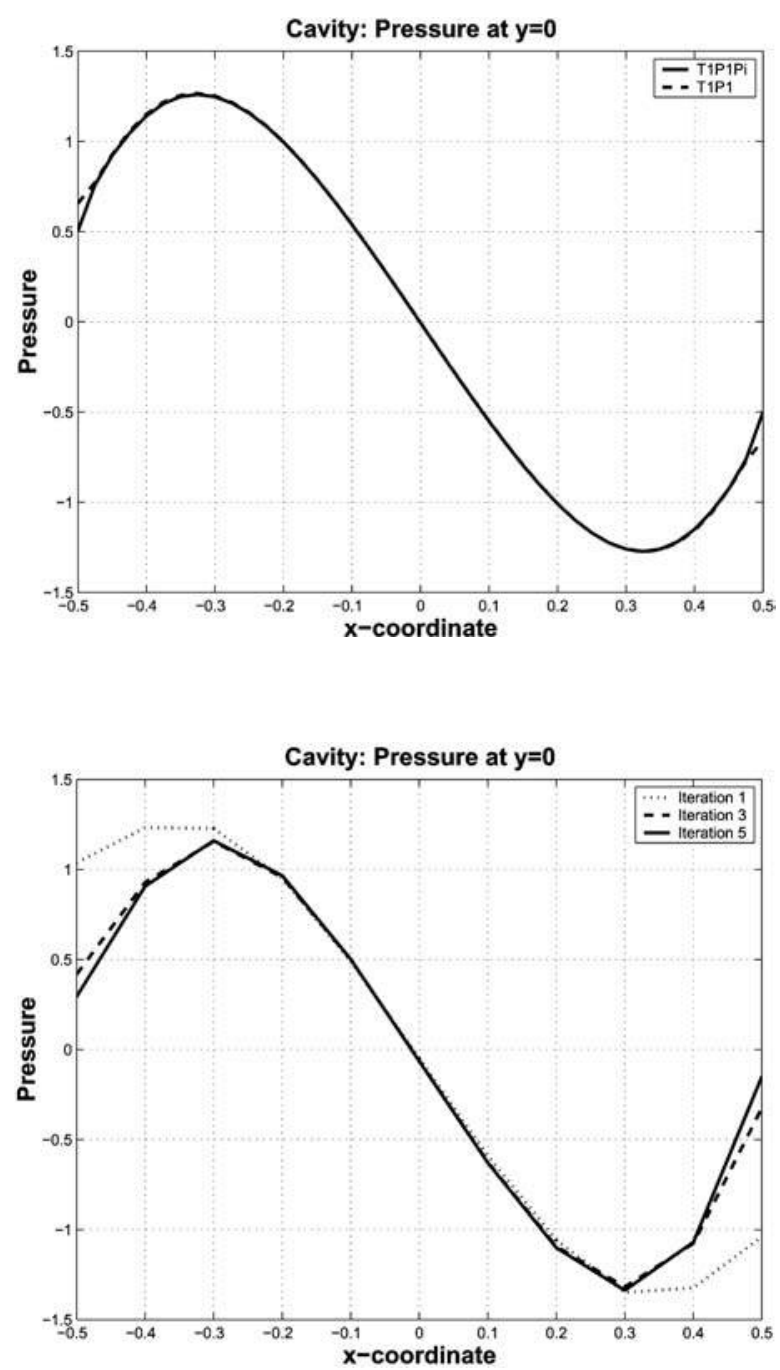

A residual correction method

651
Figure 15.

Pressure on horizontal centerline for $40 \times 40$

mesh
Figure 16.

Pressure on horizontal centerline for $10 \times 10$ mesh and iterative solution 
EC

$20,5 / 6$

652
$10 \times 10$ mesh was iterated to convergence and the behavior for the results at the upper left corner are shown in Figure 18 (note that $\Pi$ is multiplied by 1,000 to permit graphical display).

Figure 19 shows the sensitivity of the pressure at the mid height for different values of the parameter $\gamma$. It is evident that much less dependence on this value results from the addition of the added $\Pi_{i}$ stabilization terms.
Figure 17.

Pressure on horizontal centerline for $40 \times 40$ mesh and iterative solution
Figure 18.

Convergence of pressure $p$ and $\Pi$ at upper left corner vs iteration number
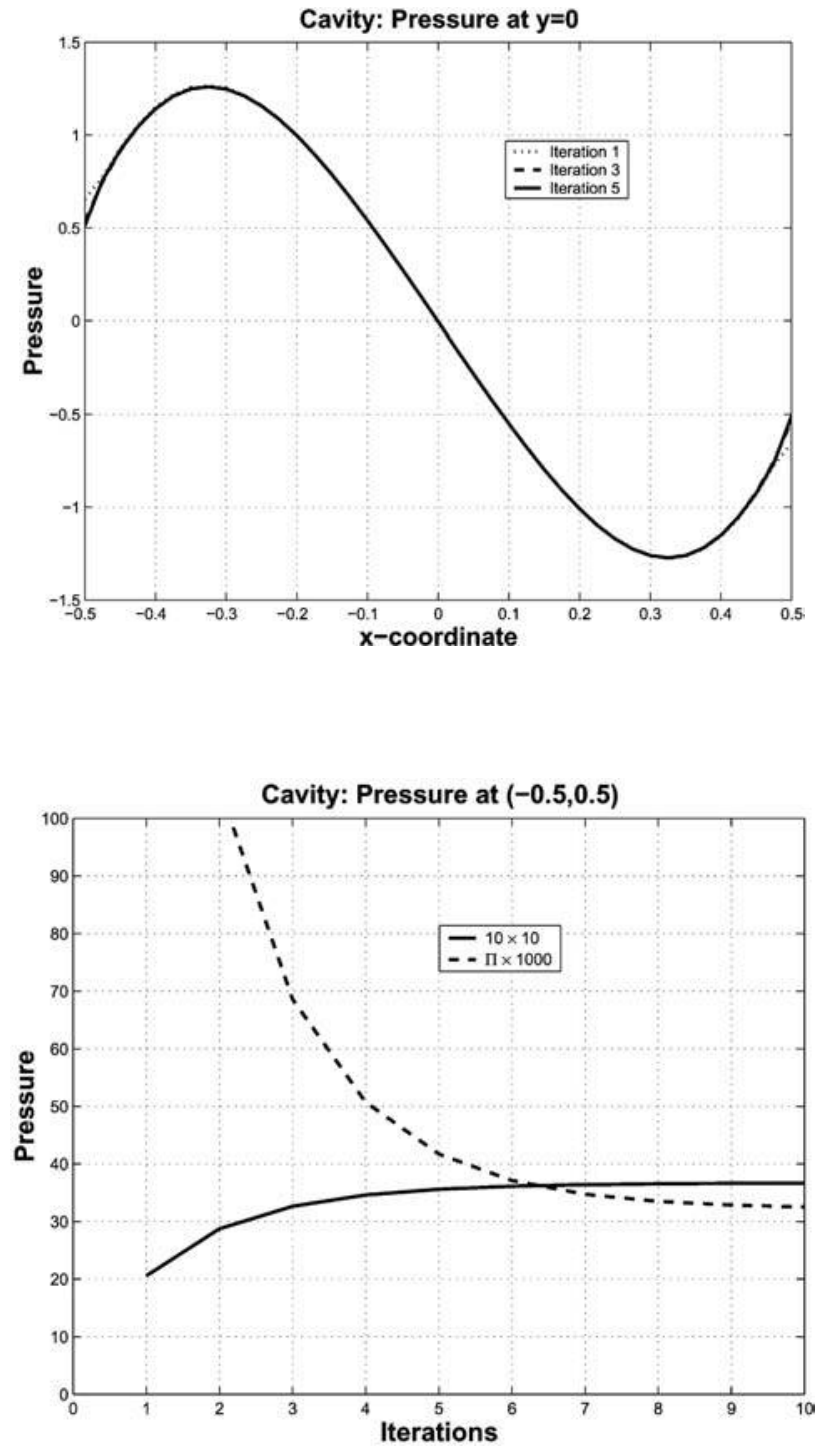


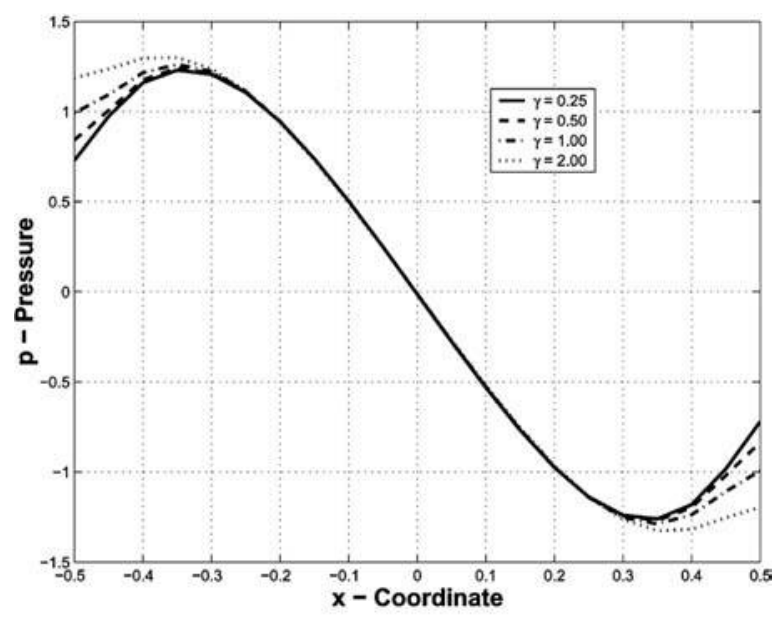

(a) Solution without $\Pi$

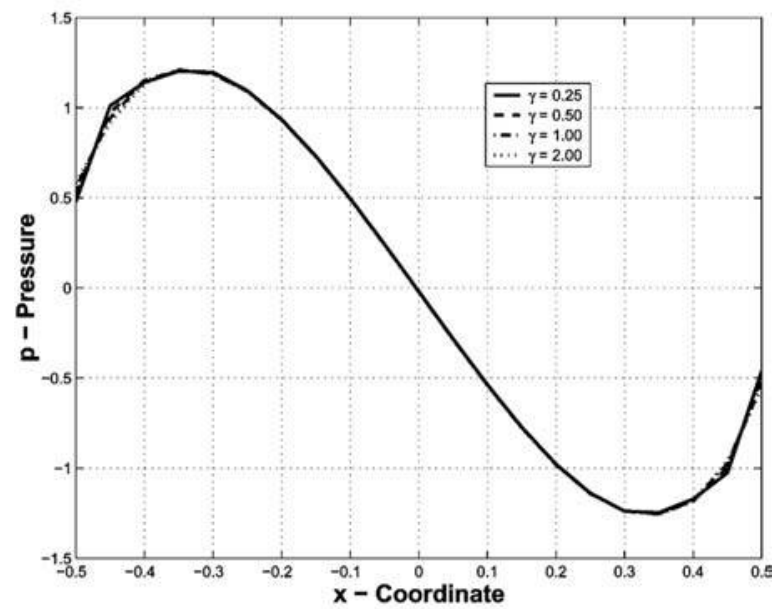

(b) Solution with $\Pi$
A residual correction method

653
Figure 19.

Dependence of pressure on value of $\gamma$ for $20 \times 20$ mesh

Finally, in Figures 20-22, the convergence of the centerline displacements and pressure are presented for different uniform mesh divisions with 10,20,40 and 80 elements per side. Again, it is evident that much less sensitivity results from the addition of the $\Pi_{i}$ terms. 
EC

$20,5 / 6$

\section{4}

Figure 20.

Dependence of

u-displacement at $x=0$ with mesh subdivision

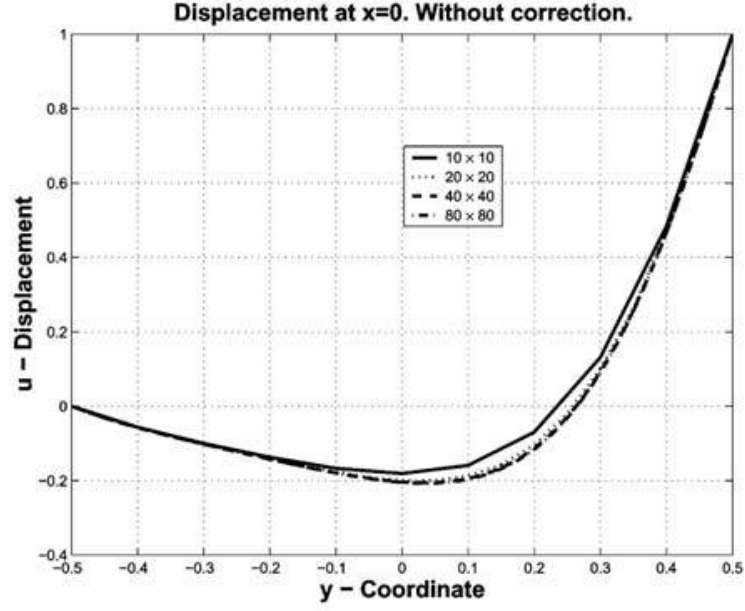

(a) Solution without $\Pi$

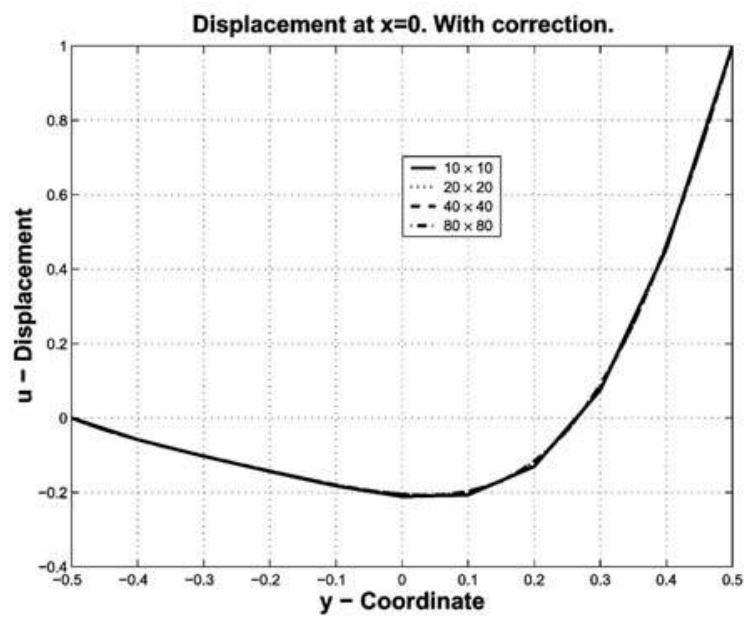

(b) Solution with $\Pi$

\section{Concluding remarks}

The concept of finite calculus which accepts that the governing equations in mechanics are satisfied in a domain of finite size, provides a natural procedure for introducing residual corrections into the discrete forms of the classical equations of the infinitesimal theory. This allows to derive new families of 


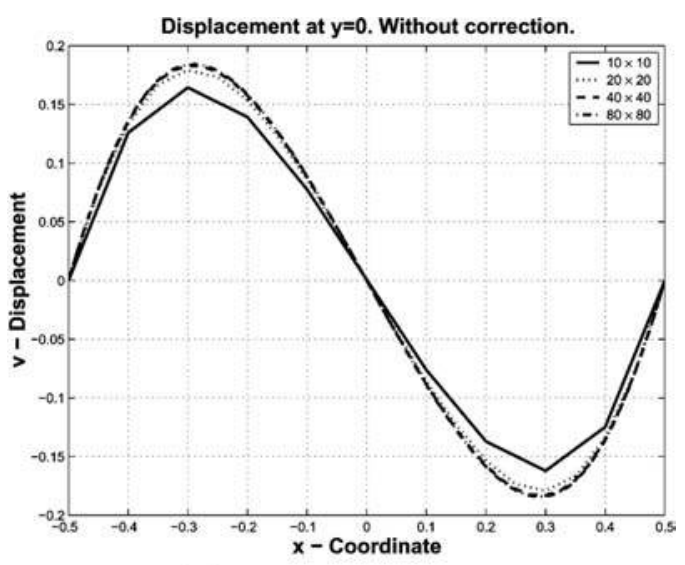

(a) Solution without $\Pi$

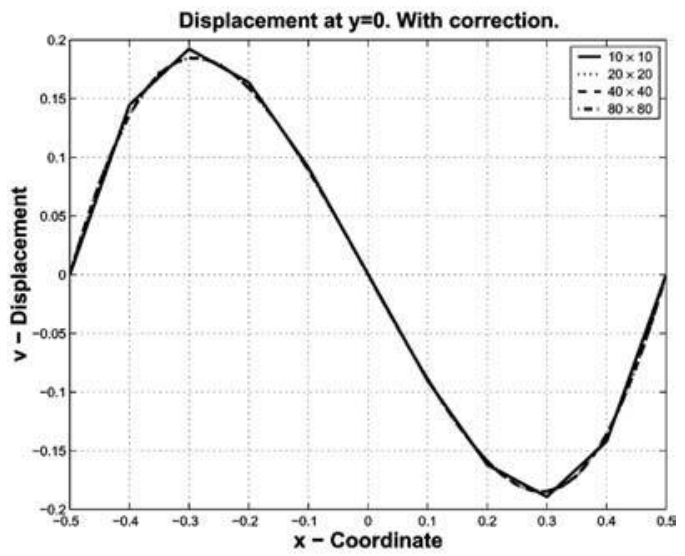

(b) Solution with $\Pi$
A residual correction method

655
Figure 21.

Dependence of $v$-displacement at $y=0$ with mesh subdivision

stabilized numerical methods using finite element, finite differences or, indeed, any other discretization procedure. In this paper, we have shown two examples of the derivation of residual corrected forms for the advective-diffusive equation and the incompressible and near (quasi) incompressible linear elasticity equations.

It is shown that for the elasticity problem, the finite calculus process results in the addition of a Laplacian of pressure terms and a projected gradient of pressure forms. The examples of application show that this form is essential to obtain accurate numerical results which converge in a 
EC

20,5/6

\section{6}

Figure 22.

Dependence of pressure at $y=0$ with mesh subdivision

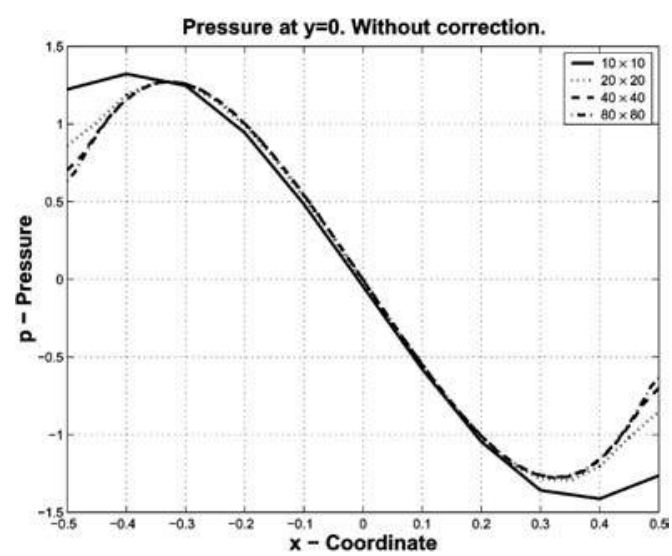

(a) Solution without $\Pi$

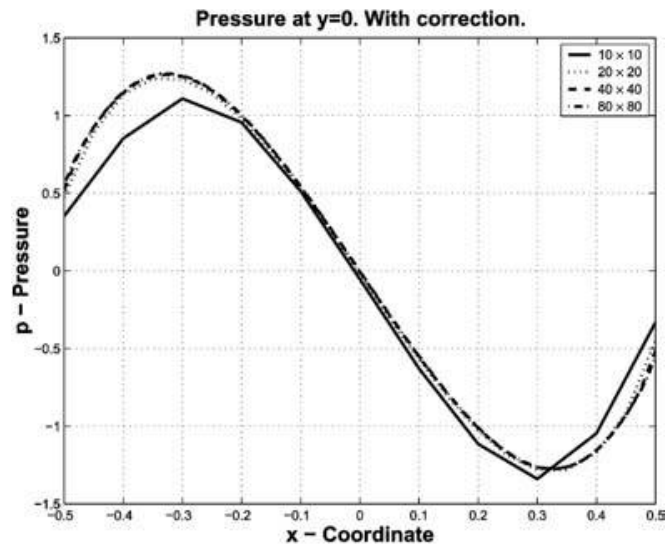

(b) Solution with $\Pi$

more monotonic manner and are less sensitive to the value of the stabilization parameters.

\section{References}

Babuška, I. (1973), "The finite element method with Lagrangian multipliers", Numer. Math., Vol. 20, pp. 179-92.

Brauchli, H.J. and Oden, J.T. (1971), "On the calculation of consistent stress distributions in finite element applications”, Int. J. Num. Meth. Eng., Vol. 3, pp. 317-25.

Brezzi, F. (1974), "On the existence, uniqueness and approximation of saddle-point problems arising from Lagrange multipliers", Rev. Française d'Automatique Inform. Rech. Opér., Ser. Rouge Anal. Numér., Vol. 8 No. R-2, pp. 129-51. 
Brezzi, F. and Pitkäranta, J. (1984), "On the stabilization of finite element approximations of the Stokes problem", in Hackbusch, W. (Ed.), Efficient Solution of Elliptic Problems, Notes on Numerical Fluid Mechanics, Vieweg, Wiesbaden, Vol. 10.

Brooks, A. and Hughes, T.J.R. (1982), "Streamline upwind/Petrov-Galerkin formulation for convection dominated flows with particular emphasis on the incompressible Navier-Stokes equations”, Comput. Meth. Appl. Mech. Eng., Vol. 32, pp. 199-259.

Cantin, G., Loubignac, C. and Touzot, C. (1978), "An iterative scheme to build continuous stress and displacement solutions”, Int. J. Num. Meth. Eng., Vol. 12, pp. 1493-506.

Chiumenti, M., Valverde, Q., Agelet de Saracibar, C. and Cervera, M. (2002a), “A stabilized formulation for incompressible elasticity using linear displacement and pressure interpolations", Comput. Meth. Appl. Mech. Eng., Vol. 191, pp. 5253-64.

Chiumenti, M., Valverde, Q., Agelet de Saracibar, C. and Cervera, M. (2002b), "A stabilized formulation for incompressible plasticity using linear triangles and tetrahedral", Int. J. Plasticity (in press).

Chorin, A.J. (1967), "A numerical method for solving incompressible viscous problems", Journal of Computational Physics, Vol. 2, pp. 12-26.

Chorin, A.J. (1968), "Numerical solution of incompressible flow problems", Studies in Numerical Analysis, Vol. 2, pp. 64-71.

Christie, I., Griffiths, D.F., Mitchell, A.R. and Zienkiewicz, O.C. (1976), "Finite element methods for second order differential equations with significant first derivatives", Int. J. Num. Meth. Eng., Vol. 10, pp. 1389-96.

Codina, R. (2000), "Stabilization of incompressibility and convection through orthogonal sub-scales in finite element methods", Comput. Meth. Appl. Mech. Eng., Vol. 190, pp. 1579-99.

Codina, R. and Blasco, J. (2000), "Stabilized finite element method for transient Navier-Stokes equations based on pressure gradient projection", Comput. Meth. Appl. Mech. Eng., Vol. 182, pp. 287-300.

Hughes, T.J.R., Franca, L.P. and Hulbert, G.M. (1989), "A new finite element formulation for computational fluid dynamics: VIII. The Galerkin/least-squares method for advective-diffusive equations", Comput. Meth. Appl. Mech. Eng., Vol. 73, pp. 173-89.

Loubibnac, C., Cantin, G. and Touzot, C. (1978), "Continuous stress fields in finite element analysis", J. AIAA, Vol. 15, pp. 1645-7.

Oñate, E. (1998), "Derivation of stabilized equations for numerical solution of advective-diffusive transport and fluid flow problems", Comput. Meth. Appl. Mech. Eng., Vol. 151, pp. 233-65.

Oñate, E. (2000), "A stabilized finite element method for incompressible flows using a finite increment calculus formulation”, Comput. Meth. Appl. Mech. Eng., Vol. 182, pp. 557-603.

Oñate, E. and Arraez, J. (2002), "Possibilities of finite calculus for deriving advanced computational methods in engineering", WCCM V Fifth World Congress on Computational Mechanics, 7-12 July 2002, Vienna.

Oñate, E. and García, J. (2001), "A finite element method for fluid-structure interaction with surface waves using a finite calculus formulation”, Comput. Meth. Appl. Mech. Eng., Vol. 191 Nos. 6/7, pp. 635-60.

Oñate, E. and Manzan, M. (1999), "A general procedure for deriving stabilized space-time finite element methods for advective-diffusive problems", International Journal for Numerical Methods in Fluids, Vol. 31, pp. 203-21.

\section{A residual correction method}

657 
$\mathrm{EC}$

$20,5 / 6$

658
Oñate, E., García, J. and Idelhson, S. (1998), "An alpha-adaptive approach for stabilized finite element solution of advective-diffusive problems", in Ladeveze, P. and Oden, J.T. (Eds), New Adv. in Adaptive Comp. Meth. in Mech., Elsevier, Amsterdam.

Oñate, E., Rojek, J., Taylor, R.L. and Zienkiewicz, O.C. (2001), "Linear triangles and tetra-hedra for incompressible problem using a finite calculus formulation", Proceedings of European Conference on Computational Mechanics, Cracow, Poland, on CD-ROM.

Oñate, E., Rojek, J., Taylor, R.L. and Zienkiewicz, O.C. (2002), "Finite calculus formulation for analysis of incompressible solids using linear triangles and tetrahedral", CIMNE Report PI 214, Int. Center for Num. Meth. Engr., May 2002, Barcelona.

Pierre, R. (1988), "Simple $C^{0}$ approximations for the computation of incompressible flows", Comput. Meth. Appl. Mech. Eng., Vol. 68, pp. 205-27.

Pierre, R. (1995), "Optimal selection of the bubble function in the stabilization of the $p_{1}-p_{1}$ element for the Stokes problem”, SIAM J. Num. Anal., Vol. 32 No. 4, pp. 1210-24.

Simo, J.C. and Rifai, M.S. (1990), "A class of mixed assumed strain methods and the method of incompatible modes”, Int. J. Num. Meth. Eng., Vol. 29, pp. 1595-638.

Simo, J.C., Taylor, R.L. and Pister, K.S. (1985), "Variational and projection methods for the volume constraint in finite deformation plasticity", Comput. Meth. Appl. Mech. Eng., Vol. 51, pp. 177-208.

Taylor, R.L. (2003), FEAP - A Finite Element Analysis Program, User Manual, University of California, Berkeley, http://www.ce.berkeley.edu/ rlt

Zienkiewicz, O.C. and Codina, R. (1995), "A general algorithm for compressible and incompressible flow - Part I: the split, characteristic-based scheme", International Journal for Numerical Methods in Fluids, Vol. 20, pp. 869-85.

Zienkiewicz, O.C. and Taylor, R.L. (1997), "The finite element patch test revisited: A computer test for convergence, validation and error estimates", Comput. Meth. Appl. Mech. Eng., Vol. 149, pp. 523-44.

Zienkiewicz, O.C. and Taylor, R.L. (2000a), The Finite Element Method: Fluid Mechanics, 5th ed., Butterworth-Heinemann, Oxford, Vol. 3.

Zienkiewicz, O.C. and Taylor, R.L. (2000b), The Finite Element Method: The Basis, 5th ed., Butterworth-Heinemann, Oxford, Vol. 1.

Zienkiewicz, O.C. and Taylor, R.L. (2000c), The Finite Element Method: Solid Mechanics, 5th ed., Butterworth-Heinemann, Oxford, Vol. 2.

Zienkiewicz, O.C. and Zhu, J.Z. (1992), "The superconvergent patch recovery (SPR) and adaptive finite element refinement”, Comput. Meth. Appl. Mech. Eng., Vol. 101, pp. 207-24.

Zienkiewicz, O.C., Li, X.K. and Nakazawa, S. (1985a), "Iterative solution of mixed problems and stress recovery procedures”, Commun. Appl. Num. Meth., Vol. 1, pp. 3-9.

Zienkiewicz, O.C., Heinrich, J.C., Huyakorn, P.S. and Mitchel, A.R. (1977), "An upwind finite element scheme for two dimensional convective transport equations", Int. J. Num. Meth. Eng., Vol. 11, pp. 131-44.

Zienkiewicz, O.C., Morgan, K., Satya Sai, B.V.K., Codina, R. and Vasquez, M. (1995), "A general algorithm for compressible and incompressible flow - Part II: tests on the explicit form", Int. J. Num. Meth. Fluids, Vol. 20, pp. 887-913.

Zienkiewicz, O.C., Qu, S., Taylor, R.L. and Nakazawa, S. (1986), “The patch test for mixed formulations", Int. J. Num. Meth. Eng., Vol. 23, pp. 1873-83.

Zienkiewicz, O.C., Vilotte, J.P., Toyoshima, S. and Nakazawa, S. (1985b), "Iterative method for constrained and mixed approximation. An inexpensive improvement of FEM performance", Comput. Meth. Appl. Mech. Eng., Vol. 51, pp. 3-29. 


\section{This article has been cited by:}

1. Ignasi de-Pouplana, Eugenio Oñate. 2018. Finite element modelling of fracture propagation in saturated media using quasi-zero-thickness interface elements. Computers and Geotechnics 96, 103-117. [Crossref]

2. Carlos A. Felippa, Eugenio Oñate, Sergio R. Idelsohn. 2017. Variational Framework for FIC Formulations in Continuum Mechanics: High Order Tensor-Derivative Transformations and Invariants. Archives of Computational Methods in Engineering 39. . [Crossref]

3. Eugenio Oñate, Prashanth Nadukandi, Juan Miquel. 2017. Accurate FIC-FEM formulation for the multidimensional steady-state advection-diffusion-absorption equation. Computer Methods in Applied Mechanics and Engineering . [Crossref]

4. Oñate Eugenio. 2016. Finite increment calculus (FIC): a framework for deriving enhanced computational methods in mechanics. Advanced Modeling and Simulation in Engineering Sciences 3:1. . [Crossref]

5. Ignasi de-Pouplana, Eugenio Oñate. 2016. A FIC-based stabilized mixed finite element method with equal order interpolation for solid-pore fluid interaction problems. International Journal for Numerical and Analytical Methods in Geomechanics . [Crossref]

6. H. Feng, X.Y. Cui, G.Y. Li. 2016. A stable nodal integration method with strain gradient for static and dynamic analysis of solid mechanics. Engineering Analysis with Boundary Elements 62, 78-92. [Crossref]

7. E. Oñate, J. Miquel, P. Nadukandi. 2016. An accurate FIC-FEM formulation for the $1 \mathrm{D}$ advectiondiffusion-reaction equation. Computer Methods in Applied Mechanics and Engineering 298, 373-406. [Crossref]

8. Eugenio Oñate, Alessandro Franci, Josep M. Carbonell. 2014. Lagrangian formulation for finite element analysis of quasi-incompressible fluids with reduced mass losses. International Journal for Numerical Methods in Fluids 74:10, 699-731. [Crossref]

9. V. Ruas, R.C.P. Leal-Toledo, M. Kischinhevsky. 2013. Elementos finitos em formulação mista de mínimos quadrados para a simulação da convecção-difusão em regime transiente. Revista Internacional de Métodos Numéricos para Cálculo y Diseño en Ingeniería 29:1, 21-28. [Crossref]

10. Jian-Hui Liao, Zhuo Zhuang. 2012. A consistent projection-based SUPG/PSPG XFEM for incompressible two-phase flows. Acta Mechanica Sinica 28:5, 1309-1322. [Crossref]

11. Eugenio Oñate, Sergio R. Idelsohn, Carlos A. Felippa. 2011. Consistent pressure Laplacian stabilization for incompressible continua via higher-order finite calculus. International Journal for Numerical Methods in Engineering 87:1-5, 171-195. [Crossref]

12. Eugenio Oñate, Prashanth Nadukandi, Sergio R. Idelsohn, Julio García, Carlos Felippa. 2011. A family of residual-based stabilized finite element methods for Stokes flows. International Journal for Numerical Methods in Fluids 65:1-3, 106-134. [Crossref]

13. Prashanth Nadukandi, Eugenio Oñate, Julio Garcia. 2008. Analysis of a consistency recovery method for the $1 \mathrm{D}$ convection-diffusion equation using linear finite elements. International Journal for Numerical Methods in Fluids 57:9, 1291-1320. [Crossref]

14. N.J. Rodriguez, K. Davey, J.L.S. Gaytan. 2007. The control volume formulation to model the convectivediffusive unsteady heat transfer over the 1-D semi-infinite domain. Computer Methods in Applied Mechanics and Engineering 196:41-44, 4059-4074. [Crossref]

15. E. Oñate, A. Valls, J. García. 2007. Computation of turbulent flows using a finite calculus-finite element formulation. International Journal for Numerical Methods in Fluids 54:6-8, 609-637. [Crossref] 
16. Eugenio Oñate, Aleix Valls, Julio García. 2007. Modeling incompressible flows at low and high Reynolds numbers via a finite calculus-finite element approach. Journal of Computational Physics 224:1, 332-351. [Crossref]

17. Eugenio Oñate, Juan Miquel, Francisco Zárate. 2007. Stabilized solution of the multidimensional advection-diffusion-absorption equation using linear finite elements. Computers \& Fluids 36:1, 92-112. [Crossref]

18. C. A. Felippa, E. Oñate. 2006. Nodally exact Ritz discretizations of 1D diffusion-absorption and Helmholtz equations by variational FIC and modified equation methods. Computational Mechanics 39:2, 91-111. [Crossref]

19. E. Oñate, J. Rojek, M. Chiumenti, S.R. Idelsohn, F. Del Pin, R. Aubry. 2006. Advances in stabilized finite element and particle methods for bulk forming processes. Computer Methods in Applied Mechanics and Engineering 195:48-49, 6750-6777. [Crossref]

20. E. Oñate, A. Valls, J. García. 2006. FIC/FEM Formulation with Matrix Stabilizing Terms for Incompressible Flows at Low and High Reynolds Numbers. Computational Mechanics 38:4-5, 440-455. [Crossref]

21. Eugenio Oñate, Juan Miquel, Guillermo Hauke. 2006. Stabilized formulation for the advection-diffusionabsorption equation using finite calculus and linear finite elements. Computer Methods in Applied Mechanics and Engineering 195:33-36, 3926-3946. [Crossref]

22. Jerzy Rojek, Eugenio Oñate, Robert L. Taylor. 2006. CBS-based stabilization in explicit solid dynamics. International Journal for Numerical Methods in Engineering 66:10, 1547-1568. [Crossref]

23. E. Oñate, J. García, S.R. Idelsohn, F. Del Pin. 2006. Finite calculus formulations for finite element analysis of incompressible flows. Eulerian, ALE and Lagrangian approaches. Computer Methods in Applied Mechanics and Engineering 195:23-24, 3001-3037. [Crossref]

24. Andrzej Truty, Thomas Zimmermann. 2006. Stabilized mixed finite element formulations for materially nonlinear partially saturated two-phase media. Computer Methods in Applied Mechanics and Engineering 195:13-16, 1517-1546. [Crossref]

25. Eugenio Oñate, Francisco Zárate, Sergio R. Idelsohn. 2006. Finite element formulation for convectivediffusive problems with sharp gradients using finite calculus. Computer Methods in Applied Mechanics and Engineering 195:13-16, 1793-1825. [Crossref] 